\title{
Generic absoluteness and boolean names for elements of a Polish space
}

\author{
Andrea Vaccaro, Matteo Viale
}

\begin{abstract}
It is common knowledge in the set theory community that there exists a duality relating the commutative $C^{*}$-algebras with the family of B-names for complex numbers in a boolean valued model for set theory $V^{\mathrm{B}}$. Several aspects of this correlation have been considered in works of the late 1970's and early 1980's, for example by Takeuti [2, 10], and by Jech [5]. Generalizing Jech's results, we extend this duality so as to be able to describe the family of boolean names for elements of any given Polish space $Y$ (such as the complex numbers) in a boolean valued model for set theory $V^{\mathrm{B}}$ as a space $C^{+}(X, Y)$ consisting of functions $f$ whose domain $X$ is the Stone space of $\mathrm{B}$, and whose range is contained in $Y$ modulo a meager set. We also outline how this duality can be combined with generic absoluteness results in order to analyze, by means of forcing arguments, the theory of $C^{+}(X, Y)$. MSC: $03 \mathbf{E 5 7}$
\end{abstract}

\section{INTRODUCTION}

There has been in the early eighties and in the late seventies a wave of attention to the possible applications of the forcing machinery in the study of certain type of operator algebras, key references are Jech's [5], and Takeuti's [2, 10]. This paper aims to revive this line of research, which in our eyes deserves more attention, at least from the set theory community. Takeuti and Jech's works outline a correspondence existing between the theory of commutative unital $C^{*}$-algebras, a specific domain of functional analysis, and the theory of Boolean valued models, which pertains to logic and set theory. Takeuti's works [2, 10] show that one can employ the general machinery of forcing to derive certain properties for spaces of operators: this is done first by interpreting these operators as suitable B-names for complex numbers in a $\mathrm{B}$-valued model for set theory $V^{\mathrm{B}}$, then showing that certain properties can be proved for these B-names using the boolean semantics for $V^{\mathrm{B}}$, finally pulling back these properties from the B-names to the corresponding operators. Jech's [5] develops an algebraic theory of commutative spaces of normal (possibly unbounded) operators, in his terminology the stonean algebras, and proves that the B-names for complex numbers in the boolean valued model for set theory $V^{\mathrm{B}}$ can be used to classify up to isomorphism all possible complete stonean algebras. He further develops a functional calculus for stonean algebras and shows that all the familiar tools given by Gelfand transform for commutative $C^{*}$-algebras naturally extend to the framework of stonean algebras. In particular [5] brings to an explicit mathematical form the duality existing between the theory of commutative $C^{*}$-algebras and the B-names for complex numbers in the boolean valued models for set theory of the form $V^{\mathrm{B}}$.

This paper generalizes this duality to arbitrary Polish spaces. We will expand on Jech's and Takeuti's works and devise a natural translation process to recast the arguments which are used to analyze the properties of real numbers in a forcing extension, into arguments that can be expressed in the language of functional analysis enriched with a tiny bit of first 
order logic. For example our results show how to transform generic absoluteness results, such as Shoenfield's absoluteness and Woodin's proof of the invariance of the theory of $L(\mathbb{R})$ under set forcing in the presence of class many Woodin cardinals [7, 14, 15, in tools to describe the degree of elementarity between the field of complex numbers (enriched eventually with Borel predicates) and the ring of germs at points of the spectrum of a commutative $C^{*}$-algebra with extremally disconnected spectrum. In particular our results can be seen as a further enhancement of the program launched by Takeuti in [2, 10] aiming to employ forcing methods in the study of operator algebras. The major outcome of the present paper can be summarized in the following definitions and result:

Let $X$ be an extremally disconnected compact Hausdorff space and $Y$ be any Polish space 1 with a Polish compactification $\bar{Y}$ : for example if $Y=\mathbb{C}, \bar{Y}=$ $\mathbb{C} \cup\{\infty\}=\mathbb{S}^{2}$ can be the one point compactification of $\mathbb{C}$.

Let $C^{+}(X, Y)$ be the space of continuous functions $f: X \rightarrow \bar{Y}$ such that the preimage of $\bar{Y} \backslash Y$ is meager in $X$ : for example if $Y=\mathbb{C}, \bar{Y}=\mathbb{C} \cup\{\infty\}=\mathbb{S}^{2}$, we require that $f^{-1}[\{\infty\}]$ is closed nowhere dense in $X$.

For any $p \in X$, let $C^{+}(X, Y) / p$ be the ring of germs in $p$ of functions in $C^{+}(X, Y)$ : i.e. $f, g \in C^{+}(X, Y)$ define the same equivalence class or germ in $C^{+}(X, Y) / p$ if $f\lceil U=g\lceil U$ for some open neighborhood $U$ of $p$.

Given $R \subseteq Y^{n}$ any Borel predicate and $p \in X$, define $R^{X} / p\left(\left[f_{1}\right], \ldots\left[f_{n}\right]\right)$ to hold if there is a neighborhood $U$ of $p$ such that $R\left(f_{1}(x), \ldots, f_{n}(x)\right)$ holds on a comeager subset of $U$. Equivalently $R^{X} / p$ is the quotient in $p$ of the boolean predicate $R^{X}: C^{+}(X, Y)^{n} \rightarrow \mathrm{CL}(X)$ mapping

$$
\left(f_{1}, \ldots, f_{n}\right) \mapsto \operatorname{Reg}\left(\left\{x \in X: R\left(f_{1}(x), \ldots, f_{n}(x)\right)\right\}\right)
$$

where $\mathrm{CL}(X)$ is the boolean algebra given by clopen subsets of $X$, and $\operatorname{Reg}(A)$ is the interior of the closure of $A$ for any $A \subseteq X$.

In essence we have defined a sheaf structure on the space of functions $C^{+}(X, Y)$, which is making sense not only of the ring of germs at any point of $X$, but also of the interpretation of the Borel predicate $R$ in such rings of germs. For example, $R$ can be the equality relation on $Y$ or the graph of multiplication on $\mathbb{C}$, or any finite (or countable) combination of such kind of Borel relations on $Y$.

We have the following theorem relating the first order theory of the rings of germs $C^{+}(X, Y) / p$ so defined, to the space $Y$ :

Theorem 1. Let $X$ be an extremally disconnected compact Hausdorff space and $Y$ be any Polish space. Fix Borel relations $R_{1} \subseteq Y^{n_{1}}, \ldots, R_{k} \subseteq Y^{n_{k}}$.

Then for all $p \in X$, the first order structure $\left\langle Y, R_{1}, \ldots, R_{n}\right\rangle$ is $\Sigma_{2}$-elementary in the first order structure $\left\langle C^{+}(X, Y) / p, R_{1}^{X} / p, \ldots, R_{n}^{X} / p\right\rangle$.

Moreover, if we assume the existence of class many Woodin cardinals, we get that

$$
\left\langle Y, R_{1}, \ldots, R_{n}\right\rangle \prec\left\langle C^{+}(X, Y) / p, R_{1}^{X} / p, \ldots, R_{n}^{X} / p\right\rangle .
$$

\footnotetext{
${ }^{1} X$ is extremally disconnected if the closure of an open set is open, or equivalently if its regular open sets are closed. $Y$ is Polish if it is a separable topological space whose topology can be induced by a complete metric on $Y$.

${ }^{2}$ Recall that $A \subseteq X$ is meager if it is the union of countably many nowhere dense sets, and $B$ is comeager in $U$ if $U \backslash B$ is meager. It requires an argument based on the fact that $R$ is Borel to show that $R^{X} / p$ is well defined.
} 
Contrary to the case of Jech's and Takeuti's works (which require also a certain degree of familiarity with the basic theory of operator algebras), our results can be understood by any reader which has familiarity with the forcing method and with the basic topological properties of Polish spaces. In the case $Y=\mathbb{C}$, the space of functions $C^{+}(X, Y)$ we consider is the unique complete stonean algebra (according to Jech's terminology of [5]) whose algebra of projections is given by the characteristic functions of clopen sets on $X$. Using Jech's methods, $C^{+}(X, \mathbb{C})$ can be described as the result of a natural limit process over the commutative and unital $C^{*}$-algebra $C(X)$. It can be seen that one can obtain a different proof of Theorem 1 for the case $Y=\mathbb{C}$ using the results of [5]. We remark nonetheless that the methods in Jech's paper do not seem to be of use if one aims to give a proof of Theorem 1 for a Polish space $Y$ other than $\mathbb{C}$ or $\mathbb{R}$, since his arguments exploit also algebraic features peculiar to the field structure of $\mathbb{C}$ and to the ordered field $\mathbb{R}$, while our arguments are purely rooted in the topological properties common to all Polish spaces.

We organize the paper as follows: in section 2 we introduce the space of functions $C^{+}(X, Y)$ with $X$ compact, Hausdorff and extremally disconnected, $Y$ Polish, and we outline its simplest properties. In section 3 we introduce the notion of B-valued model for a first order signature, and we show how to endow $C^{+}(X, Y)$ of the structure of a B-valued model for $\mathrm{B}$ the boolean algebra given by regular open (or clopen) sets of $X$. In section 4 we exhibit a natural isomorphism existing between the B-valued models $C^{+}(X, Y)$ (for B the clopen sets of $X$ ) and the family of boolean names for elements of the Polish space $Y$ as computed in the boolean valued model for set theory $V^{\mathrm{B}}$. In section 5 we show how to translate generic absoluteness results in a proof of Theorem 1, This paper outlines the original parts of the master thesis of the first author [11. A thorough presentation of all the results (and the missing details) presented here can be found there. We try to make the statements of the theorems comprehensible to most readers with a fair acquaintance with first order logic. On the other hand the proofs of our main results will require a great familiarity with the forcing method. We encounter a problem in the exposition: those familiar with forcing arguments will find most of the proofs redundant or trivial, those unfamiliar with forcing will find the paper far too sketchy. We aim to address readers of both kinds, so the current presentation tries to cope with this tension at the best of our possibilities.

\section{The space of functions $C^{+}(S t(\mathrm{~B}))$}

We refer the reader to [11, Chapter 2] or to [3, Chapter 10] for a detailed account on the material presented in this section. Let $(X, \tau)$ be a topological space. For $A \subseteq X$, the interior of $A \operatorname{lnt}(A)$ is the union of all open sets contained in $A$ and the closure of $A \mathrm{Cl}(A)$ is the intersection of all closed sets containing $A$. $A$ is regular open if it coincides with the interior of its closure. $\operatorname{Reg}(A)=\operatorname{lnt}(\mathrm{Cl}((A)))$ is the regular open set we attach to any $A \subseteq X$.

- A topological space $(X, \tau)$ is 0-dimensional if its clopen sets form a basis for $\tau$.

- A compact topological space $(X, \tau)$ is extremally (extremely) disconnected if its algebra of clopen sets $\mathrm{CL}(X)$ coincides with its algebra of regular open sets $\mathrm{RO}(X)$.

For a boolean algebra B, we let $S t(\mathrm{~B})$ be the Stone space of its ultrafilters with topology generated by the clopen sets

$$
\mathcal{O}_{b}=\{G \in S t(\mathrm{~B}): b \in G\} .
$$

The following holds: 
- $S t(\mathrm{~B})$ is a compact 0-dimensional Hausdorff space, and any 0-dimensional compact Hausdorff space $(X, \tau)$ is isomorphic to $\operatorname{St}(\mathrm{CL}(X))$,

- A compact Hausdorff space $(X, \tau)$ is extremally disconnected if and only if its algebra of clopen sets is a complete boolean algebra. In particular $S t(\mathrm{~B})$ is extremally disconnected if and only if $\mathrm{B}=\mathrm{CL}(\operatorname{St}(\mathrm{B}))$ is complete.

Recall also that the algebra of regular open sets of a topological space $(X, \tau)$ is always a complete boolean algebra with operations

- $\bigvee\left\{A_{i}: i \in I\right\}=\operatorname{Reg}\left(\bigcup\left\{A_{i}: i \in I\right\}\right)$,

- $\neg A=\operatorname{lnt}(X \backslash A)$,

- $A \wedge B=A \cap B$.

An antichain on a boolean algebra $\mathrm{B}$ is a subset $A$ such that $a \wedge b=0_{\mathrm{B}}$ for all $a, b \in A$, $\mathrm{B}^{+}=\mathrm{B} \backslash\left\{0_{\mathrm{B}}\right\}$ is the family of positive elements of $\mathrm{B}$, and a dense subset of $\mathrm{B}^{+}$is a subset $D$ such that for all $b \in \mathrm{B}^{+}$there is $a \in D$ such that $a \leq_{\mathrm{B}} b$. In a complete boolean algebra $\mathrm{B}$ any dense subset $D$ of $\mathrm{B}^{+}$contains an antichain $A$ such that $\bigvee A=\bigvee D=1_{\mathrm{B}}$, recall also that a predense subset $X$ of $\mathrm{B}$ is a subset such that $\bigvee X=1_{\mathrm{B}}$ or equivalently such that its downward closure is dense in $\mathrm{B}^{+}$.

Another key observation on Stone spaces of complete boolean algebras we will often need is the following:

Fact 2.1. Assume $\mathrm{B}$ is a complete atomless boolean algebra, then on its Stone space St(B):

- $\mathcal{O}_{\bigvee_{\mathrm{B}} A}=\operatorname{Reg}\left(\bigcup_{a \in A} \mathcal{O}_{a}\right)$ for all $A \subseteq \mathrm{B}$.

- $\mathcal{O}_{\bigvee_{\mathrm{B}} A}=\bigcup_{a \in A} \mathcal{O}_{a}$ for all finite sets $A \subseteq \mathrm{B}$.

- For any infinite antichain $A \subseteq \mathrm{B}^{+}, \bigcup_{a \in A} \mathcal{O}_{a}$ is properly contained in $\mathcal{O}_{\bigvee_{\mathrm{B}} A}$ as a dense open subset $(\{\neg a: a \in A\}$ has the finite intersection property and can be extended to an ultrafilter disjoint from $A)$.

Given a compact Hausdorff topological space $X$, we let $C^{+}(X)$ be the space of continuous functions

$$
f: X \rightarrow \mathbb{S}^{2}=\mathbb{C} \cup\{\infty\}
$$

(where $\mathbb{S}^{2}$ is seen as the one point compactification of $\mathbb{C}$ ) with the property that $f^{-1}[\{\infty\}]$ is a closed nowhere dense (i.e. with a dense open complement) subset of $X$. In this manner we can endow $C^{+}(X)$ of the structure of a commutative ring of functions with involution, letting the operations be defined pointwise on all points whose image is in $\mathbb{C}$, and be undefined on the preimage of $\infty$. More precisely $f+g$ is the unique continuous function

$$
h: X \rightarrow \mathbb{S}^{2}
$$

such that $h(x)=f(x)+g(x)$ whenever this makes sense (it makes sense on an open dense subset of $X$, since the preimage of the point at infinity under $f, g$ is closed nowhere dense) and is extended by continuity on the points on which $f(x)+g(x)$ is undefined. Thus $f+g \in C^{+}(X)$ if $f, g \in C^{+}(X)$. Similarly we define the other operations. We take the convention that constant functions are always denoted by their constant value, and that $0=1 / \infty$. We leave to the reader as an instructive exercise the following: 
Lemma 2.2. Let $X$ be compact Hausdorff extremally disconnected. Then for any $p \in X$ the ring of germs $C^{+}(X) / p$ is an algebraically closed field.

Its proof will be an immediate corollary of the main theorem we stated in the introduction, since the theory of algebraically closed fields is axiomatizable by means of $\Pi_{2}$-formulas using simple Borel predicates on $\mathbb{C}^{n}$ for all $n$. However, as a warm up for the sequel, the reader can try to prove that it is a field.

Remark 2.3. The reader is averted that the spaces of functions $C^{+}(X)$ we are considering may not be exotic: for example let $\nu$ denote the Lebesgue measure on $\mathbb{R}$, and MALG denote the complete boolean algebra given by Lebesgue-measurable sets modulo Lebesgue null sets. $C(S t(\mathrm{MALG}))$ is isometric to $L^{\infty}(\mathbb{R})$ via the Gelfand-transform of the $C^{*}$-algebra $L^{\infty}(\mathbb{R})$, and consequently $S t$ (MALG) is homeomorphic to the space of characters of $L^{\infty}(\mathbb{R})$ endowed with the weak-* topology inherited from the dual of $L^{\infty}(\mathbb{R}) . C^{+}(\operatorname{St}(\mathrm{MALG}))=$ $L^{\infty+}(\mathbb{R})$ is obtained by adding to $L^{\infty}(\mathbb{R})$ the measurable functions $f: \mathbb{R} \rightarrow \mathbb{S}^{2}$ such that $\nu\left(\bigcap_{n \rightarrow \infty}\{x:|f(x)|>n\}\right)=0$.

Moreover by means of the Gelfand transform the spaces $C^{+}(X)$ we are considering are always obtained canonically from a commutative unital $C^{*}$-algebra with extremally disconnected spectrum by a completion procedure as the one described above for $L^{\infty+}(\mathbb{R})$. Jech's [5] is an useful source for those aiming to explore further this analogy.

\section{Boolean Valued Models}

In a first order model a formula can be interpreted as true or false. Given a complete boolean algebra B, B-boolean valued models generalize Tarski semantics associating to each formula a value in $B$, so that there are no more only true and false propositions (those associated to $1_{B}$ and $0_{B}$ respectively), but also other "intermediate values" of truth. The classic definition of boolean valued models for set theory and of their semantic for the language $\mathcal{L}=\{\in\}$ may be found in [4, Chapter 14]. As mentioned earlier, we need to generalize the definition to any first order language and to any theory of the language. A complete account of the theory of these boolean valued models can be found in $[9$. Since this book is a bit out of date, we recall below the basic facts we will need and we invite the reader to consult [11, Chapter 3] for a detailed account on the material of this section.

Definition 3.1. Given a complete boolean algebra B and a first order language

$$
\mathcal{L}=\left\{R_{i}: i \in I\right\} \cup\left\{f_{j}: j \in J\right\}
$$

a B-boolean valued model (or B-valued model) $\mathcal{M}$ in the language $\mathcal{L}$ is a tuple

$$
\left\langle M,=^{\mathcal{M}}, R_{i}^{\mathcal{M}}: i \in I, f_{j}^{\mathcal{M}}: j \in J\right\rangle
$$

where:

1. $M$ is a non-empty set, called domain of the B-boolean valued model, whose elements are called B-names;

2. $={ }^{\mathcal{M}}$ is the boolean value of the equality:

$$
\begin{aligned}
={ }^{\mathcal{M}}: M^{2} & \rightarrow \mathrm{B} \\
(\tau, \sigma) & \mapsto \llbracket \tau=\sigma \rrbracket_{\mathrm{B}}^{\mathcal{M}}
\end{aligned}
$$


3. The forcing relation $R_{i}^{\mathcal{M}}$ is the boolean interpretation of the $n$-ary relation symbol $R_{i}$ :

$$
\begin{aligned}
R_{i}^{\mathcal{M}}: M^{n} & \rightarrow \mathrm{B} \\
\left(\tau_{1}, \ldots, \tau_{n}\right) & \mapsto \llbracket R_{i}\left(\tau_{1}, \ldots, \tau_{n}\right) \rrbracket_{\mathrm{B}}^{\mathcal{M}}
\end{aligned}
$$

4. $f_{j}^{\mathcal{M}}$ is the boolean interpretation of the $n$-ary function symbol $f_{j}$ :

$$
\begin{aligned}
f_{j}^{\mathcal{M}}: M^{n+1} & \rightarrow \mathrm{B} \\
\left(\tau_{1}, \ldots, \tau_{n}, \sigma\right) & \mapsto \llbracket f_{j}\left(\tau_{1}, \ldots, \tau_{n}\right)=\sigma \rrbracket_{\mathrm{B}}^{\mathcal{M}}
\end{aligned}
$$

We require that the following conditions hold:

for $\tau, \sigma, \chi \in M$,

(i) $\llbracket \tau=\tau \rrbracket_{\mathrm{B}}^{\mathcal{M}}=1_{\mathrm{B}}$;

(ii) $\llbracket \tau=\sigma \rrbracket_{\mathrm{B}}^{\mathcal{M}}=\llbracket \sigma=\tau \rrbracket_{\mathrm{B}}^{\mathcal{M}}$;

(iii) $\llbracket \tau=\sigma \rrbracket_{\mathrm{B}}^{\mathcal{M}} \wedge \llbracket \sigma=\chi \rrbracket_{\mathrm{B}}^{\mathcal{M}} \leq \llbracket \tau=\chi \rrbracket_{\mathrm{B}}^{\mathcal{M}}$;

for $R \in \mathcal{L}$ with arity $n$, and $\left(\tau_{1}, \ldots, \tau_{n}\right),\left(\sigma_{1}, \ldots, \sigma_{n}\right) \in M^{n}$,

(iv) $\left(\bigwedge_{h \in\{1, \ldots, n\}} \llbracket \tau_{h}=\sigma_{h} \rrbracket_{\mathrm{B}}^{\mathcal{M}}\right) \wedge \llbracket R\left(\tau_{1}, \ldots, \tau_{n}\right) \rrbracket_{\mathrm{B}}^{\mathcal{M}} \leq \llbracket R\left(\sigma_{1}, \ldots, \sigma_{n}\right) \rrbracket_{\mathrm{B}}^{\mathcal{M}} ;$

for $f_{j} \in \mathcal{L}$ with arity $n$, and $\left(\tau_{1}, \ldots, \tau_{n}\right),\left(\sigma_{1}, \ldots, \sigma_{n}\right) \in M^{n}$ and $\mu, \nu \in M$,

(v) $\left(\bigwedge_{h \in\{1, \ldots, n\}} \llbracket \tau_{h}=\sigma_{h} \rrbracket_{\mathrm{B}}^{\mathcal{M}}\right) \wedge \llbracket f_{j}\left(\tau_{1}, \ldots, \tau_{n}\right)=\mu \rrbracket_{\mathrm{B}}^{\mathcal{M}} \leq \llbracket f_{j}\left(\sigma_{1}, \ldots, \sigma_{n}\right)=\mu \rrbracket_{\mathrm{B}}^{\mathcal{M}} ;$

(vi) $\bigvee_{\mu \in M} \llbracket f_{j}\left(\tau_{1}, \ldots, \tau_{n}\right)=\mu \rrbracket_{\mathrm{B}}^{\mathcal{M}}=1_{\mathrm{B}}$;

(vii) $\llbracket f_{j}\left(\tau_{1}, \ldots, \tau_{n}\right)=\mu \rrbracket_{\mathrm{B}}^{\mathcal{M}} \wedge \llbracket f_{j}\left(\tau_{1}, \ldots, \tau_{n}\right)=\nu \rrbracket_{\mathrm{B}}^{\mathcal{M}} \leq \llbracket \mu=\nu \rrbracket_{\mathrm{B}}^{\mathcal{M}}$.

If no confusion can arise, we will omit the subscript $B$ and the superscript $\mathcal{M}$ and we will confuse a function or predicate symbol with its interpretation.

Given a B-valued model $\left\langle M,={ }^{M}\right\rangle$ for the equality, a forcing relation $R$ on $M$ is a map $R: M^{n} \rightarrow \mathrm{B}$ satisfying condition (iv) above for boolean predicates.

We now define the relevant maps between these objects.

Definition 3.2. Let $\mathcal{M}$ be a $\mathrm{B}$-valued model and $\mathcal{N}$ a $\mathrm{C}$-valued model in the same language $\mathcal{L}$. Let

$$
i: \mathrm{B} \rightarrow \mathrm{C}
$$

be a morphism of boolean algebras and $\Phi \subseteq M \times N$ a relation. The couple $\langle i, \Phi\rangle$ is a morphism of boolean valued models if:

1. $\operatorname{dom} \Phi=M$;

2. given $\left(\tau_{1}, \sigma_{1}\right),\left(\tau_{2}, \sigma_{2}\right) \in \Phi$ :

$$
i\left(\llbracket \tau_{1}=\tau_{2} \rrbracket_{\mathrm{B}}^{\mathcal{M}}\right) \leq \llbracket \sigma_{1}=\sigma_{2} \rrbracket_{\mathrm{C}}^{\mathcal{N}},
$$

3. given $R$ an $n$-ary relation symbol and $\left(\tau_{1}, \sigma_{1}\right), \ldots,\left(\tau_{n}, \sigma_{n}\right) \in \Phi$ :

$$
i\left(\llbracket R\left(\tau_{1}, \ldots, \tau_{n}\right) \rrbracket_{\mathrm{B}}^{\mathcal{M}}\right) \leq \llbracket R\left(\sigma_{1}, \ldots, \sigma_{n}\right) \rrbracket_{\mathrm{C}}^{\mathcal{N}},
$$


4. given $f$ an $n$-ary function symbol and $\left(\tau_{1}, \sigma_{1}\right), \ldots,\left(\tau_{n}, \sigma_{n}\right),(\mu, \nu) \in \Phi$ :

$$
i\left(\llbracket f\left(\tau_{1}, \ldots, \tau_{n}\right)=\mu \rrbracket_{\mathrm{B}}^{\mathcal{M}}\right) \leq \llbracket f\left(\sigma_{1}, \ldots, \sigma_{n}\right)=\nu \rrbracket_{\mathrm{C}}^{\mathcal{N}},
$$

An injective morphism is a morphism such that in 2 equality holds.

An embedding of boolean valued models is an injective morphism such that in 3 and 4 equality holds.

An embedding $\langle i, \Phi\rangle$ from $\mathcal{M}$ to $\mathcal{N}$ is called isomorphism of boolean valued models if $i$ is an isomorphism of boolean algebras, and for every $b \in N$ there is $a \in M$ such that $(a, b) \in \Phi$.

Suppose $\mathcal{M}$ is a $\mathrm{B}$-valued model and $\mathcal{N}$ a $\mathrm{C}$-valued model (both in the same language $\mathcal{L})$ such that $\mathrm{B}$ is a complete subalgebra of $\mathrm{C}$ and $M \subseteq N$. Let $J$ be the immersion of $\mathcal{M}$ in $\mathcal{N} . \mathcal{N}$ is said to be a boolean extension of $\mathcal{M}$ if $\left\langle i d_{\mathrm{B}}, J\right\rangle$ is an embedding of boolean valued models.

Remark 3.3. When $\mathrm{B}=\mathrm{C}$ we will consider $i=i d_{\mathrm{B}}$ unless otherwise stated.

Since we are allowing function symbols in $\mathcal{L}$, the definition of the semantic is a bit more intricate than in the case of a purely relational language.

Definition 3.4. Given a B-valued model $\mathcal{M}$ in a language $\mathcal{L}$, let $\varphi$ be an $\mathcal{L}$-formula whose free variables are in $\left\{x_{1}, \ldots, x_{n}\right\}$, and let $\nu$ be a valuation of the free variables in $\mathcal{M}$ whose domain contains $\left\{x_{1}, \ldots, x_{n}\right\}$. We denote with $\llbracket \varphi(\nu) \rrbracket_{\mathrm{B}}^{\mathcal{M}}$ the boolean value of $\varphi(\nu)$.

First, let $t$ be an $\mathcal{L}$-term and $\tau \in M$; we define recursively $\llbracket(t=\tau)(\nu) \rrbracket_{\mathrm{B}}^{\mathcal{M}} \in \mathrm{B}$ as follows:

- if $t$ is a variable $x$, then

$$
\llbracket(x=\tau)(\nu) \rrbracket_{\mathrm{B}}^{\mathcal{M}}=\llbracket \nu(x)=\tau \rrbracket_{\mathrm{B}}^{\mathcal{M}}
$$

- if $t=f\left(t_{1}, \ldots, t_{n}\right)$ where $t_{i}$ are terms and $f$ is an $n$-ary function symbol, then

$$
\llbracket\left(f\left(t_{1}, \ldots, t_{n}\right)=\tau\right)(\nu) \rrbracket_{\mathrm{B}}^{\mathcal{M}}=\bigvee_{\sigma_{1}, \ldots, \sigma_{n} \in M}\left(\bigwedge_{1 \leq i \leq n} \llbracket\left(t_{i}=\sigma_{i}\right)(\nu) \rrbracket_{\mathrm{B}}^{\mathcal{M}}\right) \wedge \llbracket f\left(\sigma_{1}, \ldots, \sigma_{n}\right)=\tau \rrbracket_{\mathrm{B}}^{\mathcal{M}}
$$

Given a formula $\varphi$, we define recursively $\llbracket \varphi(\nu) \rrbracket_{\mathrm{B}}^{\mathcal{M}}$ as follows:

- if $\varphi \equiv t_{1}=t_{2}$, then

$$
\llbracket\left(t_{1}=t_{2}\right)(\nu) \rrbracket_{\mathrm{B}}^{\mathcal{M}}=\bigvee_{\tau \in M} \llbracket\left(t_{1}=\tau\right)(\nu) \rrbracket_{\mathrm{B}}^{\mathcal{M}} \wedge \llbracket\left(t_{2}=\tau\right)(\nu) \rrbracket_{\mathrm{B}}^{\mathcal{M}}
$$

- if $\varphi \equiv R\left(t_{1}, \ldots, t_{n}\right)$, then

$$
\llbracket\left(R\left(t_{1}, \ldots, t_{n}\right)\right)(\nu) \rrbracket_{\mathrm{B}}^{\mathcal{M}}=\bigvee_{\tau_{1}, \ldots, \tau_{n} \in M}\left(\bigwedge_{1 \leq i \leq n} \llbracket\left(t_{i}=\tau_{i}\right)(\nu) \rrbracket_{\mathrm{B}}^{\mathcal{M}}\right) \wedge \llbracket R\left(\tau_{1}, \ldots, \tau_{n}\right) \rrbracket_{\mathrm{B}}^{\mathcal{M}}
$$

- if $\varphi \equiv \neg \psi$, then

$$
\llbracket \varphi(\nu) \rrbracket_{\mathrm{B}}^{\mathcal{M}}=\neg \llbracket \psi(\nu) \rrbracket_{\mathrm{B}}^{\mathcal{M}}
$$


- if $\varphi \equiv \psi \wedge \theta$, then

$$
\llbracket \varphi(\nu) \rrbracket_{\mathrm{B}}^{\mathcal{M}}=\llbracket \psi(\nu) \rrbracket_{\mathrm{B}}^{\mathcal{M}} \wedge \llbracket \theta(\nu) \rrbracket_{\mathrm{B}}^{\mathcal{M}}
$$

- if $\varphi \equiv \exists y \psi(y)$, then

$$
\llbracket \varphi(\nu) \rrbracket_{\mathrm{B}}^{\mathcal{M}}=\bigvee_{\tau \in M} \llbracket \psi(y / \tau, \nu) \rrbracket_{\mathrm{B}}^{\mathcal{M}}
$$

If no confusion can arise, we omit the index $\mathcal{M}$ and the subscript $\mathrm{B}$, and we simply denote the boolean value of a formula $\varphi$ with parameters in $\mathcal{M}$ by $\llbracket \varphi \rrbracket$.

By definition, an isomorphism of boolean valued models preserves the boolean value of the atomic formulas. Proceeding by induction on the complexity, one can get the result for any formula.

Proposition 3.5. Let $\mathcal{M}$ be a $\mathrm{B}$-valued model and $\mathcal{N}$ a $\mathrm{C}$-valued model in the same language $\mathcal{L}$. Assume $\langle i, \Phi\rangle$ is an isomorphism of boolean valued models. Then for any $\mathcal{L}$-formula $\varphi\left(x_{1}, \ldots, x_{n}\right)$, and for every $\left(\tau_{1}, \sigma_{1}\right), \ldots,\left(\tau_{n}, \sigma_{n}\right) \in \Phi$ we have that:

$$
i\left(\llbracket \varphi\left(\tau_{1}, \ldots, \tau_{n}\right) \rrbracket_{\mathrm{B}}^{\mathcal{M}}\right)=\llbracket \varphi\left(\sigma_{1}, \ldots, \sigma_{n}\right) \rrbracket_{\mathrm{C}}^{\mathcal{N}}
$$

With elementary arguments it is possible to prove the Soundness Theorem also for boolean valued models.

Theorem 3.6 (Soundness Theorem). Assume that $\varphi$ is an $\mathcal{L}$-formula which is syntactically provable by an $\mathcal{L}$-theory $T$, and that each formula in $T$ has boolean value at least $b \in \mathrm{B}$ in a $\mathrm{B}$-valued model $\mathcal{M}$. Then $\llbracket \varphi(\nu) \rrbracket_{\mathrm{B}}^{\mathcal{M}} \geq b$ for all valuations $\nu$ in $\mathcal{M}$.

We get a first order model from a B-valued model passing to a quotient by an ultrafilter $G \subseteq \mathrm{B}$. This corresponds for spaces of type $C^{+}(S t(\mathrm{~B}))$ to a specialization of the space to the ring of germs in $G$. In the general context it is defined as follows.

Definition 3.7. Let B a complete boolean algebra, $\mathcal{M}$ a B-valued model in the language $\mathcal{L}$, and $G$ an ultrafilter over B. Consider the following equivalence relation on $M$ :

$$
\tau \equiv_{G} \sigma \Leftrightarrow \llbracket \tau=\sigma \rrbracket \in G
$$

The first order model $\mathcal{M} / G=\left\langle M / G,={ }^{\mathcal{M}} / G, R_{i}^{\mathcal{M} / G}: i \in I, f_{j}^{\mathcal{M} / G}: j \in J\right\rangle$ is defined letting:

- For any $n$-ary relation symbol $R$ in $\mathcal{L}$

$$
R^{\mathcal{M} / G}=\left\{\left(\left[\tau_{1}\right]_{G}, \ldots,\left[\tau_{n}\right]_{G}\right) \in(M / G)^{n}: \llbracket R\left(\tau_{1}, \ldots, \tau_{n}\right) \rrbracket \in G\right\} .
$$

- For any $n$-ary function symbol $f$ in $\mathcal{L}$

$$
\begin{aligned}
f^{\mathcal{M} / G}:(M / F)^{n} & \rightarrow M / G \\
\left(\left[\tau_{1}\right]_{G}, \ldots,\left[\tau_{n}\right]_{G}\right) & \mapsto[\sigma]_{G} .
\end{aligned}
$$

where $\sigma$ is such that $\llbracket f\left(\tau_{1}, \ldots, \tau_{n}\right)=\sigma \rrbracket \in G$. Def. 3.1(vii) guarantees that this function is well defined.

If we require $\mathcal{M}$ to satisfy a key additional condition, we get an easy way to control the truth value of formulas in $\mathcal{M} / G$. 
Definition 3.8. A B-valued model $\mathcal{M}$ for the language $\mathcal{L}$ is full if for every $\mathcal{L}$-formula $\varphi(x, \vec{y})$ and every $\vec{\tau} \in M^{|\vec{y}|}$ there is a $\sigma \in M$ such that

$$
\llbracket \exists x \varphi(x, \vec{\tau}) \rrbracket=\llbracket \varphi(\sigma, \vec{\tau}) \rrbracket
$$

Theorem 3.9 (Boolean Valued Models Łoś's Theorem). Assume $\mathcal{M}$ is a full B-valued model for the language $\mathcal{L}$. Then for every formula $\varphi\left(x_{1}, \ldots, x_{n}\right)$ in $\mathcal{L}$ and $\left(\tau_{1}, \ldots, \tau_{n}\right) \in$ $M^{n}$ :

(i) For all ultrafilters $G$ over $\mathrm{B}, \mathcal{M} / G \models \varphi\left(\left[\tau_{1}\right]_{G}, \ldots,\left[\tau_{n}\right]_{G}\right)$ if and only if $\llbracket \varphi\left(\tau_{1}, \ldots, \tau_{n}\right) \rrbracket \in$ $G$.

(ii) For all $a \in \mathrm{B}$ the following are equivalent:

(a) $\llbracket \varphi\left(f_{1}, \ldots, f_{n}\right) \rrbracket \geq a$,

(b) $\mathcal{M} / G \models \varphi\left(\left[\tau_{1}\right]_{G}, \ldots,\left[\tau_{n}\right]_{G}\right)$ for all $G \in \mathcal{O}_{a}$,

(c) $\mathcal{M} / G \models \varphi\left(\left[\tau_{1}\right]_{G}, \ldots,\left[\tau_{n}\right]_{G}\right)$ for densely many $G \in \mathcal{O}_{a}$.

\section{1. $C^{+}(S t(\mathrm{~B}))$ as a boolean valued extension of $\mathbb{C}$}

The following example shows how to obtain a boolean extension of a topological space $Y$ for a language composed by symbols which are interpreted as Borel subsets of $Y^{n}$.

Example 3.10. Fix a complete boolean algebra B and a topological space $Y$ such that

$$
\Delta_{Y}=\{(x, x) \in Y \times Y: x \in Y\}
$$

is Borel on $Y \times Y$. Consider $M=C(S t(\mathrm{~B}), Y)$, the set of continuous functions from $S t(\mathrm{~B})$ to $Y$.

We define a structure of B-valued extension of $Y$ on $M$ for the language with equality as follows. Given $f, g \in M$, the set

$$
W=\{G \in S t(\mathrm{~B}): f(G)=g(G)\}
$$

is a Borel subset of $S t(\mathrm{~B})$ since both $f$ and $g$ are continuous. Recall that $A \subseteq Y$ is meager if it is contained in a countable union of closed nowhere dense sets, and that $A$ has the Baire property if $U \Delta A$ is meager for some (unique) regular open set $U$. Since every Borel set $B$ has the Baire property [4, Lemma 11.15], and $S t(\mathrm{~B})$ is compact Hausdorff, by [3, Chapter 29, Lemma 5], we get that

$$
\operatorname{Reg}(\{G \in \operatorname{St}(\mathrm{B}): f(G)=g(G)\})
$$

is the unique regular open set with a meager symmetric difference with $W$. Identifying $\mathrm{B}$ with $\mathrm{RO}(S t(\mathrm{~B}))$ (B is complete), we have that

$$
={ }^{S t(\mathrm{~B})}(f, g)=\llbracket f=g \rrbracket^{S t(\mathrm{~B})}=\operatorname{Reg}(\{G \in S t(\mathrm{~B}): f(G)=g(G)\})
$$

is a well defined element of B and satisfies the clauses of Def. 3.1 for the equality relation. For any Borel $R \subseteq Y^{n}$, the predicate $R^{S t(\mathrm{~B})}: C(S t(\mathrm{~B}), Y)^{n} \rightarrow \mathrm{B}$ defined by

$$
R^{S t(\mathrm{~B})}\left(f_{1}, \ldots, f_{n}\right)=\llbracket R\left(f_{1}, \ldots, f_{n}\right) \rrbracket^{S t(\mathrm{~B})}=\operatorname{Reg}\left(\left\{G \in S t(\mathrm{~B}): R\left(f_{1}(G), \ldots, f_{n}(G)\right)\right\}\right)
$$

is a forcing relation $R$ satisfying the clauses of Def. 3.1. Similarly we can lift Borel functions $F: Y^{n} \rightarrow Y$. 
With these definitions it can be checked that

$$
\mathcal{M}=\left\langle C(S t(\mathrm{~B}), Y),={ }^{S t(\mathrm{~B})}, R_{i}^{S t(\mathrm{~B})}: i \in I, F_{j}^{S t(\mathrm{~B})}: j \in J\right\rangle
$$

is a $\mathrm{B}$-valued model for the signature given by the Borel relations $\left\langle R_{i}: i \in I\right\rangle$ and Borel functions $\left\langle F_{j}: j \in J\right\rangle$ chosen on $Y$. Moreover the set $\left\{c_{x} \in M: x \in Y\right\}$, where $c_{x}$ is the constant function with value $x$, is a copy of $Y$ in $M$, i.e: the complete homomorphism given by the inclusion of 2 in $\mathrm{B}$ induces an embedding of the 2 -valued model $\left\langle Y,=, R_{i}\right.$ : $\left.i \in I, F_{j}: j \in J\right\rangle$ into the B-valued model $\mathcal{M}$ mapping $x \mapsto c_{x}$ (however we do not as yet assert that this embedding preserves the truth of formulas with quantifiers). Thus we can infer that $\mathcal{M}$ is a B-valued extension of an isomorphic copy of $Y$ seen as a 2-valued structure in a relational language with relation symbols interpreted as Borel subsets of $Y^{n}$.

Finally if $G$ is an ultrafilter on $S t(\mathrm{~B})$, i.e. a point of $S t(\mathrm{~B})=X$, we can define the ring $C(X, Y) / G$ of germs in $C(X, Y)$ letting

$$
[f]_{G}=\left\{g: \llbracket f=g \rrbracket^{S t(\mathrm{~B})} \in G\right\}
$$

and $\left.R^{S t(\mathrm{~B})}\left(\left[f_{1}\right]_{G}, \ldots,\left[f_{n}\right]_{G}\right]\right)$ iff $R^{S t(\mathrm{~B})}\left(f_{1}, \ldots, f_{n}\right) \in G$. We can easily check that the map $x \mapsto\left[c_{x}\right]_{G}$ defines an embedding of 2 -valued models of $\left\langle Y,=, R_{i}: i \in I, F_{j}: j \in J\right\rangle$ into $\mathcal{M} / G$.

If $Y$ is Polish (i.e. second countable and completely metrizable) $\Delta_{Y}$ is closed ( $Y$ is Hausdorff), therefore, for any fixed language $\mathcal{L}$ whose elements are Borel relations and functions on $Y$, we can define a structure of B-valued extension of $Y$ for the language $\mathcal{L}$. If $Y=\mathbb{C}$, the domain of such extension is the $C^{*}$-algebra $C(S t(\mathrm{~B}))$ with extremally disconnected spectrum.

It can be checked that if $Y$ is compact, $C(S t(\mathrm{~B}), Y)$ endowed with suitable lifting of Borel predicates is a full B-valued model, while if $Y$ is not compact and contains an infinite set with discrete relative topology (e.g. $\mathbb{N}$ as a subset of $\mathbb{C}), C(S t(\mathrm{~B}), Y)$ is not a full B-valued model (see Remark 4.4 below).

The latter observation is one of the compelling reasons which lead us to associate to $\mathbb{C}$ (which is Polish non-compact, locally compact) the space of functions $C^{+}(\operatorname{St}(\mathrm{B})$ ) (which we show to be a full B-valued model). Similar tricks will be needed to properly describe the full boolean extensions of arbitrary (non-compact) Polish spaces by means of spaces of functions.

We resume the above observations in the following definition:

Definition 3.11. Let $X$ be a compact Hausdorff extremally disconnected topological space.

(i) Let $Y$ be a topological space such that $\Delta_{Y}$ is Borel in $Y^{2}$. For any Borel relation $R$ on $Y^{n}, R^{X}: C(X, Y)^{n} \rightarrow \mathrm{CL}(X)$ maps $\left(f_{1}, \ldots, f_{n}\right)$ to the clopen set

$$
\operatorname{Reg}\left(\left\{G \in X: R\left(f_{1}(G), \ldots, f_{n}(G)\right)\right\}\right) .
$$

The lifting of Borel functions on $Y$ to $C(X, Y)$ is obtained by lifting their graph to a forcing relation on $C(X, Y)$.

(ii) We let $C^{+}(X)$ be the space of continuous functions

$$
f: X \rightarrow \mathbb{S}^{2}=\mathbb{C} \cup\{\infty\}
$$


(where $\mathbb{S}^{2}$ is seen as the one point compactification of $\mathbb{C}$ ) with the property that $f^{-1}[\{\infty\}]$ is a closed nowhere dense subset of $X$. We lift Borel relations $R \subseteq \mathbb{C}^{n}$ to $R^{X}$ again letting

$$
R^{X}\left(f_{1}, \ldots, f_{n}\right)=\operatorname{Reg}\left(\left\{G \in X: R\left(f_{1}(G), \ldots, f_{n}(G)\right)\right\}\right) .
$$

We let $\left\langle C(X) / G, R^{X} / G\right\rangle$ and $\left\langle C^{+}(X) / G, R^{X} / G\right\rangle$ be the associated ring of germs with $R^{X} / G$ defined for both rings by the requirement: $\left.R^{X}\left(\left[f_{1}\right]_{G}, \ldots,\left[f_{n}\right]_{G}\right]\right)$ iff $G \in R^{X}\left(f_{1}, \ldots, f_{n}\right)$.

We have the following Lemmas:

Lemma 3.12 (Mixing Lemma). Assume $\mathrm{B}$ is a complete boolean algebra and $A \subseteq \mathrm{B}$ is an antichain. Then for all families $\left\{f_{a}: a \in A\right\} \subseteq C^{+}(\operatorname{St}(\mathrm{B}))$, there exists $f \in C^{+}(\operatorname{St}(\mathrm{B}))$ such that

$$
a \leq \llbracket f=f_{a} \rrbracket
$$

for all $a \in A$.

Proof. Sketch: Let $f \in C^{+}(S t(\mathrm{~B}))$ be the unique function such that $f\left\lceil\mathcal{O}_{(\neg \vee A)}=0\right.$ and $f \uparrow \mathcal{O}_{a}=f_{a} \uparrow \mathcal{O}_{a}$ for all $a \in A$. Check that $f$ is well defined and works.

Lemma 3.13 (Fullness Lemma). Assume $\mathrm{B}$ is a complete boolean algebra. Let $R_{1}, \ldots, R_{n}$ be forcing relations on $C^{+}(S t(\mathrm{~B}))^{<\mathbb{N}}$. Then for all formulas $\varphi(x, \vec{y})$ in the language $\left\{R_{1}, \ldots, R_{n}\right\}$ and all $\vec{f} \in C^{+}(S t(\mathrm{~B}))^{n}$, there exists $g \in C^{+}(S t(\mathrm{~B}))$ such that

$$
\llbracket \exists x \varphi(x, \vec{f}) \rrbracket=\llbracket \varphi(g, \vec{f}) \rrbracket .
$$

Proof. Sketch: Find $A$ maximal antichain among the $b$ such that $\llbracket \varphi\left(g_{b}, \vec{f}\right) \rrbracket \geq b$ for some $g_{b}$. Now apply the Mixing Lemma to patch together all the $g_{a}$ for $a \in A$ in a $g$. Check that

$$
\llbracket \exists x \varphi(x, \vec{f}) \rrbracket=\llbracket \varphi(g, \vec{f}) \rrbracket .
$$

\section{B-NAMES FOR ElEments of A POLISh SPACE}

We refer the reader to [4] for a comprehensive treatment of the forcing method, and to [11, Chapter 3] for a sketchy presentation covering in more detail the results of this section. All over this section we assume the reader has some familiarity with the standard presentations of forcing and we follow notation standard in the set theoretic community (for example $\mathbb{N}$ is often denoted as the ordinal $\omega$ ). Throughout this section we will assume $V$ (the universe of sets) to be a transitive model of ZFC, and B $\in V$ a boolean algebra which $V$ models to be complete. $V^{\mathrm{B}}$ will denote the boolean valued model of set theory as defined in [4, Chapter 14] and $\check{a} \in V^{\mathrm{B}}$ will denote the canonical B-names for sets $a \in V$. If $G$ is a $V$-generic ultrafilter in B, $V[G]$ will denote the generic extension of $V$ and $\sigma_{G}$ the interpretations of B-names in $V^{\mathrm{B}}$ by $G$. In this situation there is a natural isomorphism between $\left(V^{\mathrm{B}} / G, \in^{\mathrm{B}} / G\right)$ and $(V[G], \in)$ defined by $[\sigma]_{G} \mapsto \sigma_{G}$. Cohen's forcing theorem in this setting states the following for any formula $\varphi\left(x_{1}, \ldots, x_{n}\right)$ in the language of set theory:

- $V[G] \models \varphi\left(\left(\sigma_{1}\right)_{G}, \ldots,\left(\sigma_{n}\right)_{G}\right)$ if and only if $\llbracket \varphi\left(\sigma_{1}, \ldots, \sigma_{n}\right) \rrbracket \in G$, 
- $\llbracket \varphi\left(\sigma_{1}, \ldots, \sigma_{n}\right) \rrbracket \geq b$ if and only if $V[G] \models \varphi\left(\left(\sigma_{1}\right)_{G}, \ldots,\left(\sigma_{n}\right)_{G}\right)$ for all $V$-generic filters $G$ to which $b$ belongs.

It is well known that $V$-generic filters cannot exist for atomless complete boolean algebra, nonetheless there is a wide spectrum of solutions to overcome this issue and work as if for any such algebra $V$-generic filters can be found, and we will do so. We will also use in several points the following form of absoluteness for $\Delta_{1}$-properties.

Let $c$ denote a new constant symbol. Then for all provably $\Delta_{1}$-definable properties $\varphi(x, c)$ over the theory ZFC $+(c \subseteq \omega)$ and all $a \in H_{\omega_{1}}$, the following holds:

- $\varphi(x / a, c / r)$ holds in a transitive $N$ which is a model of (a large enough fragment of) ZFC with $a, r \in N$ if and only if $\llbracket \varphi(x / \check{a}, c / \check{r}) \rrbracket=1_{\mathrm{B}}$ holds in $N$ for all boolean algebras $\mathrm{B} \in N$ which $N$ models to be complete.

- $\varphi(x / a, c / r)$ holds in $V$ if and only if it holds in any (some) transitive set $N$ which is a model of (a large enough fragment of) ZFC with $a, r \in N$.

Let $Y$ be a Polish space. Then $Y$ can be identified with a $G_{\delta}$-subset of the Hilbert cube $\mathcal{H}=[0,1]^{\mathbb{N}}[\underline{6}$, Theorem 4.14].

Consider

$$
\hat{\mathcal{B}}=\left\{B_{r}(q): r \in \mathbb{Q}, q \in D\right\}
$$

where $B_{r}(q)$ is the open ball of radius $r$ and center $q$, and $D$ is the set of points in $\mathcal{H}$ with rational coordinates which are non-zero just on a finite set. Then $\hat{\mathcal{B}}$ is a countable basis for the topology on $\mathcal{H}=[0,1]^{\mathbb{N}}$, and it is described by a provably $\Delta_{1}$-definable property defined by a lightface Borel predicate.

Definition 4.1. Let $Y$ be a Polish space in $V$, w.l.o.g.

$$
Y=\bigcap_{n \in \mathbb{N}} \bigcup\left\{B_{r_{m n}}\left(q_{m n}\right): m \in \mathbb{N}\right\}
$$

is a $G_{\delta}$-subset of $\mathcal{H}$ given by a suitably chosen family of elements $B_{r_{m n}}\left(q_{m n}\right)$ of $\hat{\mathcal{B}} . \sigma \in V^{\mathrm{B}}$ is a B-name for an element of $Y$ if

$$
\llbracket \sigma \in \bigcap_{n \in \mathbb{N}} \bigcup\left\{\dot{B}_{r_{m n}}\left(q_{m n}\right): m \in \mathbb{N}\right\} \rrbracket=1_{\mathrm{B}},
$$

where $\left(\dot{B}_{r}(q)\right)_{G}$ is in $V[G]$ the ball of radius $r$ and center $q$ of the space $\mathcal{H}$ as defined in $V[G]$ for all $V$-generic filters $G$.

We denote by $Y^{\mathrm{B}}$ the set of all B-names (of minimal rank) for elements of $Y$ modulo the equivalence relation:

$$
\sigma \equiv \tau \Leftrightarrow \llbracket \sigma=\tau \rrbracket=1_{\mathrm{B}}
$$

We will call B-name for a complex number any element of the family $\mathbb{C}^{\mathrm{B}}$.

We can similarly lift Borel relations on $Y^{n}$ to boolean relations on $\left(Y^{\mathrm{B}}\right)^{n}$ :

\footnotetext{
${ }^{3}$ Recall that $H_{\omega_{1}}$ is the family of hereditarily countable sets. For what concerns us, the relevant observation is that any Polish space is a definable class (with parameters) in $H_{\omega_{1}}$.
} 
Remark 4.2. Let $Y$ be a Polish space. As already noted, $Y$ is a $G_{\delta}$-subset of $\mathcal{H}=[0,1]^{\mathbb{N}}$. $\hat{\mathcal{B}}$ induces a countable open basis on $Y$ :

$$
\hat{\mathcal{B}}_{Y}=\left\{B_{r}(q) \cap Y: r \in \mathbb{Q}, q \in D\right\} .
$$

Every Borel subset of $Y$ is obtained, in fewer than $\aleph_{1}$ steps, from the elements of $\hat{\mathcal{B}}_{Y}$ by taking countable unions and complements. It is possible to code these operations with $r$ a subset of $\omega$ (see [4, Chapter 25]). For our purposes it is enough to say that if $R$ is a Borel subset of $Y^{n}$, there is some $r \subseteq \omega$ and a (ZFC provably) $\Delta_{1}$-property $P_{R}(\vec{x}, y)$ such that

$$
\vec{x} \in R \Leftrightarrow P_{R}(\vec{x}, r) .
$$

Suppose $r \in V$. We denote by $R^{V}$ the set $\left\{\vec{x} \in V: P_{R}(\vec{x}, r)\right\}$.

Guided by these considerations, we define in $V$ the following.

Definition 4.3. Given $R$, a Borel $n$-ary relation on a Polish space $Y$, we let $P_{R}(\vec{x}, r)$ be the provably $\Delta_{1}$-definable property such that

$$
\vec{x} \in R \Leftrightarrow P_{R}(\vec{x}, r) .
$$

For any $\sigma_{1}, \ldots, \sigma_{n} \in Y^{\mathrm{B}}$, let $\vec{\sigma} \in V^{\mathrm{B}}$ denote the canonical name for the tuple $\left(\sigma_{1}, \ldots, \sigma_{n}\right)$.

Define

$$
R^{\mathrm{B}}\left(\sigma_{1}, \ldots, \sigma_{n}\right)=\llbracket P_{R}(\vec{\sigma}, \check{r}) \rrbracket^{V^{\mathrm{B}}} .
$$

Similarly define the lifting to $Y^{\mathrm{B}}$ of Borel functions $F: Y^{n} \rightarrow Y$.

With these definitions

$$
\left\langle Y^{\mathrm{B}}, R_{1}^{\mathrm{B}}, \ldots, R_{k}^{\mathrm{B}}, F_{1}^{\mathrm{B}}, \ldots, F_{l}^{\mathrm{B}}\right\rangle
$$

is a B-valued extension of $Y$, where each $R_{i}\left(F_{j}\right)$ is an arbitrary Borel relation (function) on $Y^{n_{i}}$ (from $Y^{m_{j}}$ to $Y$ ).

Remark 4.4. So far we have defined a structure of B-valued model for Borel relations and functions on both $Y^{\mathrm{B}}$ and $C(S t(\mathrm{~B}), Y)$ for a Polish space $Y$. However, whenever $Y$ is not compact, we cannot exhibit a natural isomorphism between these two models, unless we enlarge $C(S t(\mathrm{~B}), Y)$. The problem (that can be appreciated by the reader familiar with forcing) is the following: assume we split a complete atomless boolean algebra $B$ in a countable maximal antichain $A=\left\{a_{n}: n \in \omega\right\}$. Then $\bigvee_{n \in \omega} a_{n}=1_{\mathrm{B}}$ but $\bigcup_{n \in \omega} \mathcal{O}_{a_{n}}$ is just an open dense subset of $\operatorname{St}(\mathrm{B})$, as the family $\left\{\neg a_{n}: n \in \omega\right\}$ has the finite intersection property and can be extended to an ultrafilter $H$ missing the antichain $A$. Now consider for $Y=\mathbb{C}$ the function $f: G \mapsto n$ iff $a_{n} \in G$. This should naturally correspond to the B-name for a natural number

$$
\sigma_{f}=\left\{\left\langle\check{m}, a_{n}\right\rangle: m<n \in \omega\right\} .
$$

Notice also that the function is continuous on its domain since the target is a discrete subspace of $\mathbb{C}$ and the preimage of each point is clopen. Moreover this function naturally extends to a continuous function in $C^{+}(S t(\mathrm{~B})) \backslash C(S t(\mathrm{~B}))$ mapping the $G$ out of its domain to $\infty$. This shows that $C(S t(\mathrm{~B}))$ is a space of functions too small to capture all possible B-names for complex numbers. The reader who has grasped the content of this remark will find the proofs of the following Lemmas almost self-evident, however we decided to include them in full details, since at some points there are delicate issues regarding the way to formulate certain simple properties of Polish spaces in an absolute (i.e $\Delta_{1}$-definable) manner, which can be tricky for those who are not fully familiar with forcing. 
Definition 4.5. Let $Y$ be a Polish space presented as a $G_{\delta^{-}}$-subset of the Hilbert cube $\mathcal{H}=[0,1]^{\mathbb{N}}$. Let $\mathrm{B}$ be a complete boolean algebra.

$C^{+}(S t(\mathrm{~B}), Y)$ is the family of continuous functions $f: S t(\mathrm{~B}) \rightarrow \mathcal{H}$ such that $f^{-1}[\mathcal{H} \backslash Y]$ is meager in $S t(\mathrm{~B})$.

We can define a structure of B-valued extension of $Y$ over $C^{+}(S t(\mathrm{~B}), Y)$ repeating verbatim what we have done in Section 3.1 for $C(S t(\mathrm{~B}), Y)$. Everything will work smoothly since for all Borel $R \subseteq Y^{n}$ and $f_{1}, \ldots, f_{n} \in C^{+}(S t(\mathrm{~B}), Y)$, the set of $H \in S t(\mathrm{~B})$ such that $R\left(f_{1}(H), \ldots, f_{n}(H)\right)$ is not defined is always a meager subset of $S t(\mathrm{~B})$. Moreover Lemmas 3.12 and 3.13 can be recasted verbatim also for $C^{+}(S t(\mathrm{~B}), Y)$, which is therefore a full $\mathrm{B}$-valued model. We are ready to prove the following theorem.

Theorem 4.6. Let $Y$ be a Polish space and $\mathrm{B}$ a complete boolean algebra.

Then $\left\langle C^{+}(S t(\mathrm{~B}), Y),={ }^{S t}(\mathrm{~B})\right\rangle$ and $\left\langle Y^{\mathrm{B}},={ }^{\mathrm{B}}\right\rangle$ are isomorphic $\mathrm{B}$-valued models.

Since the case $Y=\mathbb{C}$ outlines already the main ingredients of the proof and may be slightly easier to follow, due to the evident analogies of the spaces $C^{+}(X, \mathbb{C})$ with commutative $C^{*}$-algebras, we will give the full proof of the theorem above for this special case. However, with minimal modifications, the reader will be able to generalize by himself the proof to any Polish space: for spaces admitting a one point compactification it suffices to replace all occurrences of $\mathbb{C}$ with $Y$ in the proof to follow. For other Polish spaces $Z$ not admitting such a simple compactification, this is slightly more delicate since the preimage of an $f \in C^{+}(S t(\mathrm{~B}), Z)$ of the points in the range of $f$ out of $Z$ is not anymore a closed nowhere dense set, but a countable union of closed nowhere dense sets of $S t(\mathrm{~B})$. However no essential new complications arise also for this case, so we feel free to sketch just the main ingredients of the proof for the more general case of such Polish spaces $Z$.

Remark 4.7. In the following, given a complete boolean algebra $\mathrm{B}$, we will often confuse it with $\operatorname{RO}(S t(\mathrm{~B}))$. If $U$ is a regular open set of $S t(\mathrm{~B})$ and $G \in S t(\mathrm{~B})$, we may write equivalently

$$
G \in U, U \in G
$$

depending on whether we are considering $U$ as an element of $\mathrm{RO}(\operatorname{St}(\mathrm{B}))$ or as the correspondent element in B.

Remark 4.8. The definitions given in Remark4.2 and Definition 4.5 can be simplified when working in $\mathbb{C}$. Instead of $\hat{\mathcal{B}}_{\mathbb{C}}$ from Remark 4.2, we will work directly with $\mathcal{B}=\left\{U_{n}: n \in \omega\right\}$, the countable basis of $\mathbb{C}$ whose elements are the open balls with rational radius and whose centre has rational coordinates. Moreover, instead of Definition 4.5, we work with $C^{+}(S t(B))$ as defined in Def. 3.11(ii).

\section{Proof of Theorem 4.6 for $\mathbb{C}$}

The proof splits in several Lemmas.

The first Lemma gives a characterization of the B-name to associate to an $f \in$ $C^{+}(S t(\mathrm{~B}))$, which we will need in order to define the boolean isomorphism we are looking for.

Lemma 4.9. Assume $f \in V$ is an element of $C^{+}(\operatorname{St}(\mathrm{B}))$. For $H \in S t(\mathrm{~B})$ we define

$$
\Sigma_{f}^{H}=\left\{\mathrm{Cl}\left(U_{n}\right): \operatorname{Reg}\left(f^{-1}\left[U_{n}\right]\right) \in H\right\}
$$

Then, for $H \in S t(\mathrm{~B})$, we have:

$$
f(H)=\sigma_{f}^{H}
$$

where $\sigma_{f}^{H}$ it is the unique element in $\bigcap \Sigma_{f}^{H}$ if $\Sigma_{f}^{H}$ is non-empty, and $\sigma_{f}^{H}=\infty$ otherwise. 
Remark 4.10. The Lemma shows that in ZFC, given $f \in C^{+}(S t(B))$, it holds that

$$
f(H)=x \Leftrightarrow x=\sigma_{f}^{H} .
$$

The latter is a (ZFC provably) $\Delta_{1}$-property with $\omega, \mathrm{B}$, and $\left\{a_{n}=\operatorname{Reg}\left(f^{-1}\left[U_{n}\right]\right): n \in \mathbb{N}\right\}$ as parameters. Thus, given $V$ a transitive model of ZFC, B a complete boolean algebra in $V, G$ a $V$-generic filter in $\mathrm{B}$, any $f \in V$ element of $C^{+}(S t(\mathrm{~B}))^{V}$ can be extended in an absolute manner to $V[G]$ by the rule:

$$
\begin{aligned}
f^{V[G]}: S t(\mathrm{~B})^{V[G]} & \rightarrow \mathbb{C}^{V[G]} \\
H & \mapsto \sigma_{f}^{H}
\end{aligned}
$$

where $\sigma_{f}^{H}$ is defined as in the previous lemma through the set $\Sigma_{f}^{H}=\left\{\mathrm{Cl}\left(U_{n}\right): a_{n} \in H\right\}$.

This observation is used in the following proposition defining the boolean isomorphism between $\mathbb{C}^{\mathrm{B}}$ and $C^{+}(S t(\mathrm{~B}))$.

Proposition 4.11. Fix $V$ a transitive model of ZFC and $\mathrm{B} \in V$ a boolean algebra which $V$ models to be complete. Let $f \in C^{+}(S t(\mathrm{~B}))$ and consider

$$
\mathcal{B}=\left\{U_{n}: n \in \omega\right\}
$$

the countable basis of $\mathbb{C}$ defined in Remark 4.8. For each $n \in \omega$ let

$$
a_{n}=\operatorname{Reg}\left(f^{-1}\left[U_{n}\right]\right) .
$$

There exists a unique $\tau_{f} \in \mathbb{C}^{\mathrm{B}}$ such that

$$
\llbracket \tau_{f} \in \dot{U}_{n} \rrbracket^{V^{\mathrm{B}}}=a_{n}
$$

for all $n \in \omega$. Moreover any $\tau \in V^{\mathrm{B}}$ such that $\llbracket \tau \in \dot{U}_{n} \rrbracket^{V^{\mathrm{B}}}=a_{n}$ for all $n \in \omega$ is also such that $\llbracket \tau=\tau_{f} \rrbracket^{V^{\mathrm{B}}}=1_{\mathrm{B}}$.

By Proposition 4.11 we conclude that the map $f \mapsto \tau_{f}$ defines a function between $C^{+}(S t(\mathrm{~B}))$ and $\mathbb{C}^{\mathrm{B}}$. We still need to show that the function is a surjective boolean map i.e. it maps boolean equality on $C^{+}(S t(\mathrm{~B}))$ to boolean equality on $\mathbb{C}^{\mathrm{B}}$ and is surjective (in the sense of boolean embeddings). The latter is achieved by the following Lemma:

Lemma 4.12. Assume $\tau \in \mathbb{C}^{\mathrm{B}}$. Consider

$$
\begin{aligned}
f_{\tau}: S t(\mathrm{~B}) & \rightarrow \mathbb{C} \cup\{\infty\} \\
H & \mapsto \sigma_{\tau}^{H}
\end{aligned}
$$

where, given

$$
\Sigma_{\tau}^{H}=\left\{\mathrm{Cl}\left(U_{n}\right): \llbracket \tau \in \dot{U}_{n} \rrbracket^{V^{\mathrm{B}}} \in H\right\}
$$

$\sigma_{\tau}^{H}$ is the unique element in $\bigcap \Sigma_{\tau}^{H}$ if $\Sigma_{f}^{H}$ is non-empty, $\sigma_{\tau}^{H}=\infty$ otherwise. The function $f_{\tau}$ belongs to $C^{+}(S t(\mathrm{~B}))$ and $\tau_{f_{\tau}}=\tau$.

\footnotetext{
${ }^{4} \dot{U}_{n}$ denotes the B-name for the complex numbers in the open ball of the generic extension determined by the rational coordinates and rational radius of the ball $U_{n}$.
} 
Finally we need to show that $f \mapsto \sigma_{f}$ respects boolean equality, i.e. that:

$$
\llbracket f=g \rrbracket^{C^{+}(S t(\mathrm{~B}))}=\llbracket \tau_{f}=\tau_{g} \rrbracket^{V^{\mathrm{B}}} .
$$

Since it makes no difference to prove the equality for this relation or for an arbitrary Borel relation (or functions), we will prove the following stronger result:

Lemma 4.13. Assume $R \subseteq \mathbb{C}^{n}$ is a Borel relation. Then $R^{S t(\mathrm{~B})}\left(f_{1}, \ldots, f_{n}\right)=R^{\mathrm{B}}\left(\sigma_{f_{1}}, \ldots, \sigma_{f_{n}}\right)$, where $R^{\mathrm{B}}$ is defined according to Def. 4.3 .

It is clear that these Lemmas entail the conclusion of the theorem. We prove all of them in the next subsection.

Corollary 4.14. Under the hypotheses of Proposition 4.11, if $G$ is a V-generic filter in $\mathrm{B}$ then:

$$
f^{V[G]}(G)=\left(\tau_{f}\right)_{G}
$$

Proof. By Lemma4.13 and Remark 4.10, we get that $\tau_{f}^{H}=f^{V[G]}(H)$ for all $H \in S t(\mathrm{~B})^{V[G]}$. Moreover whenever $H$ is $V$-generic for B we also have that $\tau_{f}^{H}=\left(\tau_{f}\right)_{H}$. Since $G \in V[G]$ is $V$-generic for $\mathrm{B}$, the conclusion follows.

\section{Proof of the key Lemmas}

Proof of Lemma 4.9. Assume $\Sigma_{f}^{H}$ is empty. If $f(H) \in U_{n}$ for some $n \in \omega$ it follows that:

$$
H \in f^{-1}\left[U_{n}\right] \subseteq \operatorname{Reg}\left(f^{-1}\left[U_{n}\right]\right)
$$

hence $\mathrm{Cl}\left(U_{n}\right) \in \Sigma_{f}^{H}$, which is absurd. Suppose now that $\Sigma_{f}^{H}$ is non-empty.

Claim 4.14.1. Assume $\Sigma_{f}^{H}$ is non-empty. Then $\bigcap \Sigma_{f}^{H}$ is a singleton.

Proof. Let $m \in \omega$ be such that $\mathrm{Cl}\left(U_{m}\right) \in \Sigma_{f}^{H}$.

Existence: The family

$$
\hat{\Sigma}_{f}^{H}=\left\{\mathrm{Cl}\left(U_{m}\right) \cap \mathrm{Cl}\left(U_{n}\right): \operatorname{Reg}\left(f^{-1}\left[U_{n}\right]\right) \in H\right\}
$$

is a family of closed subsets of $\mathrm{Cl}\left(U_{m}\right)$. $\Sigma_{f}^{H}$ inherits the finite intersection property from $H$, hence so does $\hat{\Sigma}_{f}^{H}$. Since $\mathrm{Cl}\left(U_{m}\right)$ is compact, we can conclude that

$$
\emptyset \neq \bigcap \hat{\Sigma}_{f}^{H} \subseteq \bigcap \Sigma_{f}^{H}
$$

Uniqueness: Suppose there are two different points $x, y \in \bigcap \Sigma_{f}^{H}$. There exists $p \in \omega$ such that $x \in U_{p}, y \notin \mathrm{Cl}\left(U_{p}\right)$. The last relation guarantees that $\mathrm{Cl}\left(U_{p}\right) \notin \Sigma_{f}^{H}$. Now we show that for $w \in \bigcap \Sigma_{f}^{H}, w \in U_{n}$ implies $\operatorname{Reg}\left(f^{-1}\left[U_{n}\right]\right) \in H$. Therefore $x \in U_{p}$ implies $\mathrm{Cl}\left(U_{p}\right) \in \Sigma_{f}^{H}$, which is absurd. Suppose $\operatorname{Reg}\left(f^{-1}\left[U_{p}\right]\right) \notin H$, we have that:

$$
H \in \operatorname{Reg}\left(f^{-1}\left[U_{p}\right]\right)^{c} \cap \operatorname{Reg}\left(f^{-1}\left[U_{m}\right]\right) \subseteq f^{-1}\left[\mathrm{Cl}\left(U_{m}\right) \backslash U_{p}\right]
$$

For each $z \in \mathrm{Cl}\left(U_{m}\right) \backslash U_{p}$ there exists $U_{n_{z}}$ such that

$$
z \in U_{n_{z}} \wedge x \notin \mathrm{Cl}\left(U_{n_{z}}\right)
$$


This family of open balls covers the compact space $\mathrm{Cl}\left(U_{m}\right) \backslash U_{p}$, so that there are $z_{1}, \cdots, z_{k} \in \mathrm{Cl}\left(U_{m}\right) \backslash U_{p}$ which verify the following chain of inclusions:

$$
f^{-1}\left[\mathrm{Cl}\left(U_{m}\right) \backslash U_{p}\right] \subseteq \bigcup_{1 \leq i \leq k} f^{-1}\left[U_{n_{z_{i}}}\right] \subseteq \bigcup_{1 \leq i \leq k} \operatorname{Reg}\left(f^{-1}\left[U_{n_{z_{i}}}\right]\right)
$$

There is therefore a $z_{j}$ such that $\operatorname{Reg}\left(f^{-1}\left[U_{n_{z_{j}}}\right]\right) \in H$, hence $\mathrm{Cl}\left(U_{z_{j}}\right) \in \Sigma_{f}^{H}$. This is absurd since $x \notin \mathrm{Cl}\left(U_{z_{j}}\right)$.

Suppose $f(H) \neq \sigma_{f}^{H}$ and consider two open balls $U_{1}, U_{2}$ in $\mathcal{B}$ such that

$$
\begin{gathered}
\mathrm{Cl}\left(U_{1}\right) \cap \mathrm{Cl}\left(U_{2}\right)=\emptyset \\
f(H) \in U_{1} \\
\sigma_{f}^{H} \in U_{2}
\end{gathered}
$$

It easily follows that both $\operatorname{Reg}\left(f^{-1}\left[U_{1}\right]\right)$ and $\operatorname{Reg}\left(f^{-1}\left[U_{2}\right]\right)$ are in $H$ (the second assertion can be shown along the same lines of the uniqueness proof in Claim 4.14.1). These two sets are disjoint, a contradiction follows.

The Lemma is proved.

In order to prove Proposition 4.11, we need to generalize what we have exposed in Remark 4.2 about Borel codes. In particular we need to be able to describe what is the lift of an open (closed) set of $S t(\mathrm{~B})$ to the corresponding open (closed) set in $S t(\mathrm{~B})^{V[G]}$ where $G$ is $V$-generic for B. The following can be shown starting from the clopen sets and then extending the proof to cover the case of arbitrary open or closed sets.

Fact 4.15. Let $G$ be a $V$-generic filter over B. Assume $R^{V}, S^{V}$ are two open or closed sets in $S t(\mathrm{~B})^{V}$. Then

$$
R^{V} \subseteq S^{V} \Leftrightarrow R^{V[G]} \subseteq S^{V[G]}
$$

Proof. We deal with the case for open sets, the case for closed sets is proved along the same lines. Let in $V, R=\bigcup_{i \in I} \mathcal{O}_{a_{i}}$ and $S=\bigcup_{j \in J} \mathcal{O}_{a_{j}}$. Now set in $V[G], R^{V[G]}=\bigcup_{i \in I} \mathcal{O}_{a_{i}}^{V[G]}$ and $S^{V[G]}=\bigcup_{j \in J} \mathcal{O}_{a_{j}}^{V[G]}$. Then $R \subseteq S$ holds in $V$ (or $R^{V[G]} \subseteq S^{V[G]}$ holds in $V[G]$ ) iff for all $i \in I \mathcal{O}_{a_{i}}^{V} \subseteq \bigcup_{j \in J} \mathcal{O}_{a_{j}}^{V}\left(\mathcal{O}_{a_{i}}^{V[G]} \subseteq \bigcup_{j \in J} \mathcal{O}_{a_{j}}^{V[G]}\right)$. By compactness, since $\mathcal{O}_{a_{i}}^{V}\left(\mathcal{O}_{a_{i}}^{V[G]}\right)$ is a clopen subset of $S t(\mathrm{~B})^{V}$ in $V$ (a clopen subset of $S t(\mathrm{~B})^{V[G]}$ in $V[G]$ ), there is a finite set $J_{i} \subseteq J$ such that $\mathcal{O}_{a_{i}} \subseteq \bigcup_{j \in J_{i}} \mathcal{O}_{a_{j}}$. This occurs (both in $V$ or $V[G]$ ) if and only if $a_{i} \leq \bigvee_{j \in J_{i}} a_{j}$

Now notice that for any finite set $J_{i}, V[G] \models a_{i} \leq \bigvee_{j \in J_{i}} a_{j}$ iff $V \models a_{i} \leq \bigvee_{j \in J_{i}} a_{j}$.

We get the thesis.

Proof of Proposition 4.11. Consider the B-name

$$
\Sigma_{f}=\left\{\left(\dot{U}_{n}, a_{n}\right): n \in \omega\right\},
$$

where $a_{n}=\operatorname{Reg}\left(f^{-1}\left[U_{n}\right]\right)$. Standard forcing arguments give that

$$
\llbracket \exists ! x\left(x \in \bigcap \Sigma_{f}\right) \rrbracket=1_{\mathrm{B}} .
$$

We give a proof of this equality for the sake of completeness: 
Proof of equation (2).

Claim 4.15.1. Let $G$ be a $V$-generic filter for B. Then:

$$
V[G] \models \exists ! x\left(x \in \bigcap \Sigma_{f}^{G}\right)
$$

where $\Sigma_{f}^{G}=\left\{\mathrm{Cl}\left(U_{n}\right)^{V[G]}: a_{n} \in G\right\}$.

Proof of the claim. The preimage of $\mathbb{C}$ through $f$ contains an open dense subset of $S t(\mathrm{~B})$ in $V$, hence it follows that

$$
D=\left\{a_{n}=\operatorname{Reg}\left(f^{-1}\left[U_{n}\right]\right): n \in \omega\right\} \in V
$$

is a predense subset of $\mathrm{B}^{+}$. Since $G$ is $V$-generic, $G \cap D \neq \emptyset$. Thus $a_{m} \in G$ and $\mathrm{Cl}\left(U_{m}\right)^{V[G]} \in \Sigma_{f}^{G}$ for some $m \in \omega$. The proof that $\bigcap \Sigma_{f}^{G}$ is a singleton can be carried out as in Claim 4.14.1.

The Claim holds for all $V$-generic filters $G$ for B, thus

$$
\llbracket \exists ! x\left(x \in \bigcap \Sigma_{f}\right) \rrbracket=1_{\mathrm{B}} .
$$

completing the proof of equation (2).

$V^{\mathrm{B}}$ is full, hence there is a B-name $\tau_{f}$ such that

$$
\llbracket \tau_{f} \in \bigcap \Sigma_{f} \rrbracket=1_{\mathrm{B}} .
$$

This is a B-name for a complex number. Moreover, if $\tau$ is a B-name for a complex number and

$$
\llbracket \tau \in \bigcap \Sigma_{f} \rrbracket=1_{\mathrm{B}},
$$

then, from

$$
\left(\tau_{f} \in \bigcap \Sigma_{f}\right) \wedge\left(\tau \in \bigcap \Sigma_{f}\right) \wedge\left(\exists ! x\left(x \in \bigcap \Sigma_{f}\right)\right) \rightarrow \tau=\tau_{f}
$$

it follows that:

$$
\llbracket \tau=\tau_{f} \rrbracket=1_{\mathrm{B}} .
$$

This shows that the map $f \mapsto \tau_{f}$ can be defined.

To conclude the proof of Proposition 4.11 we still must show that

$$
\llbracket \tau_{f} \in \dot{U}_{n} \rrbracket=\operatorname{Reg}\left(f^{-1}\left[U_{n}\right]\right)=a_{n}
$$

Proof of equation (3). Let $G$ be a $V$-generic filter for B. On the one hand we have (using the same proof of the uniqueness part in Claim 4.14.1) that if $\left(\tau_{f}\right)_{G} \in U_{n}^{V[G]}$ then $a_{m} \in G$ for some $m$ such that $\left(\dot{U}_{n}, a_{m}\right) \in \Sigma_{f}$ and $a_{m} \in G$, which necessarily gives that $m=n$, obtaining

$$
\llbracket \tau_{f} \in \dot{U}_{n} \rrbracket \leq a_{n} .
$$

On the other hand

$$
\left.G \in f^{V[G]-1}\left[U_{n}^{V[G]}\right] \Rightarrow\left(\tau_{f}\right)_{G}={ }^{b y} 4.14\right] f^{V[G]}(G) \in U_{n}^{V[G]} \Rightarrow \llbracket \tau_{f} \in \dot{U}_{n} \rrbracket \in G
$$


which means, interpreting $\llbracket \tau_{f} \in \dot{U}_{n} \rrbracket$ as a clopen subset of $S t(\mathrm{~B})^{V[G]}$, that

$$
f^{V[G]^{-1}}\left[U_{n}^{V[G]}\right] \subseteq\left(\llbracket \tau_{f} \in \dot{U}_{n} \rrbracket\right)^{V[G]} .
$$

Lemma 4.15 guarantees that this is equivalent to

$$
f^{-1}\left[U_{n}^{V}\right] \subseteq \llbracket \tau_{f} \in \dot{U}_{n} \rrbracket .
$$

Since $\llbracket \tau_{f} \in \dot{U}_{n} \rrbracket$ is clopen, this implies that

$$
a_{n}=\operatorname{Reg}\left(f^{-1}\left[U_{n}^{V}\right]\right) \leq \llbracket \tau_{f} \in \dot{U}_{n} \rrbracket
$$

Proposition 4.11 is proved.

Proof of Lemma 4.12. The proof that $\Sigma_{\tau}^{H}$ is non-empty iff its intersection has one single point can be carried out as in Claim 4.14.1 substituting all over the proof $\operatorname{Reg}\left(f^{-1}\left[U_{n}\right]\right)$ with $\llbracket \tau \in \dot{U}_{n} \rrbracket$.

Preimage of $\{\infty\}$ is nowhere dense: We show that the preimage of $\mathbb{C}$ through $f_{\tau}$ contains an open dense set. Set

$$
a_{n}=\llbracket \tau \in \dot{U}_{n} \rrbracket
$$

and consider the set $A=\left\{a_{n}: n \in \omega\right\}$. We show that:

$$
\bigvee_{n \in \omega} a_{n}=1_{\mathrm{B}}
$$

Since $\tau$ is a B-name for a complex number in $M$, if $G$ is a $V$-generic filter over B we have:

$$
V[G] \models \tau^{G} \in \mathbb{C}^{V[G]}
$$

We can thus infer

$$
V[G] \models \exists n \in \omega\left(\tau^{G} \in U_{n}\right)
$$

for all $V$-generic filters $G$, since $\mathbb{C}^{V[G]} \cap V[G]=\bigcup_{n \in \omega} U_{n}^{V[G]} \cap V[G]$. Thus:

$$
\bigvee_{n \in \omega} a_{n}=\llbracket \exists n \in \check{\omega}\left(\tau \in \dot{U}_{n}\right) \rrbracket \geq 1_{\mathrm{B}}
$$

This implies that $A$ is predense and therefore that $\bigcup_{n \in \omega} \mathcal{O}_{a_{n}}$ is dense in $\operatorname{St}(\mathrm{B})$.

Continuous: Let $H \in S t(\mathrm{~B})$ be in the preimage of $\mathbb{C}$, and let $U$ be an open subset of $\mathbb{C}$ containing $f_{\tau}(H)$. Consider $U_{k} \in \mathcal{B}$ such that

$$
\begin{aligned}
& f_{\tau}(H) \in U_{k} \\
& \mathrm{Cl}\left(U_{k}\right) \subseteq U
\end{aligned}
$$

Since

$$
f_{\tau}(H) \in U_{k} \Rightarrow a_{k} \in H
$$


(this can be proved as in the uniqueness part in Claim 4.14.1 substituting $\operatorname{Reg}\left(f^{-1}\left[U_{n}\right]\right)$ with $\left.\llbracket \tau \in \dot{U}_{n} \rrbracket\right)$, and since the following inclusion holds

$$
\mathcal{O}_{a_{k}} \subseteq f_{\tau}^{-1}(U)
$$

the continuity of $f_{\tau}$ for points in the preimage of $\mathbb{C}$ is proved.

Consider now $H \in f_{\tau}^{-1}(\{\infty\})$. Let $A$ be an open neighborhood of $\infty$, and let $U_{k} \in \mathcal{B}$ be such that:

$$
\mathrm{Cl}\left(U_{k}\right)^{c} \subseteq A
$$

We also consider $U_{l}$ such that

$$
\mathrm{Cl}\left(U_{k}\right) \subseteq U_{l}
$$

By definition of $f_{\tau}$ we have that $H \in \mathcal{O}_{a_{l}}^{c}$, and by equation (11) the image of any element in the open set $\mathcal{O}_{a_{l}}^{c}$ cannot belong to $U_{l}$. Thus

$$
\mathcal{O}_{a_{l}}^{c} \subseteq f_{\tau}^{-1}\left[U_{l}^{c}\right] \subseteq f_{\tau}^{-1}\left[\mathrm{Cl}\left(U_{k}\right)^{c}\right] \subseteq f_{\tau}^{-1}[A]
$$

$\underline{\tau_{f_{\tau}}=\tau:}$ We already know that (see equation (1)):

$$
f_{\tau}^{-1}\left[U_{n}\right] \subseteq \mathcal{O}_{a_{n}}
$$

The second set is clopen, therefore:

$$
\llbracket \tau_{f_{\tau}} \in \dot{U}_{n} \rrbracket=\operatorname{Reg}\left(f_{\tau}^{-1}\left[U_{n}\right]\right) \subseteq \mathcal{O}_{a_{n}}
$$

Toward a contradiction, assume $\llbracket \tau=\tau_{f_{\tau}} \rrbracket \neq 1_{\mathrm{B}}$ and let $G$ a $V$-generic filter which verifies

$$
V[G] \models \tau_{G} \neq\left(\tau_{f_{\tau}}\right)_{G}
$$

Thus there exists $n \in \omega$ such that:

$$
\begin{gathered}
\left(\tau_{f_{\tau}}\right)_{G} \in U_{n}^{V[G]} \\
\tau_{G} \notin U_{n}^{V[G]}
\end{gathered}
$$

The inclusion relation (2) implies

$$
\llbracket \tau_{f_{\tau}} \in \dot{U}_{n} \rrbracket \leq a_{n}=\llbracket \tau \in \dot{U}_{n} \rrbracket
$$

but by Cohen's Forcing Theorem $\llbracket \tau_{f_{\tau}} \in \dot{U}_{n} \rrbracket \in G$. This is a contradiction.

The Lemma is proved.

Proof of Lemma 4.13. We will consider in detail the case of $R \subseteq \mathbb{C}$ a unary Borel relation in $\mathbb{C}$, the general case for $n$-ary $R$ is immediate. Given $f \in C^{+}(S t(\mathrm{~B}))$, consider $\llbracket R(f) \rrbracket$ and $\llbracket \tau_{f} \in \dot{R} \rrbracket$ as regular open subsets of $S t(\mathrm{~B})$. In order to show that they are equal, it is sufficient to prove that their symmetric difference is meager. By definition, we already know that $\llbracket R(f) \rrbracket$ has meager difference with the set

$$
\{H \in S t(\mathrm{~B}): f(H) \in R\}=f^{-1}[R] .
$$

Therefore it suffices to prove that $\llbracket \tau_{f} \in \dot{R} \rrbracket$ and $f^{-1}[R]$ have meager difference. The proof proceeds step by step on the hierarchy of Borel sets $\Sigma_{\alpha}^{0}, \Pi_{\alpha}^{0}$, for $\alpha$ a countable ordinal. 
$\underline{\Sigma_{1}^{0}}$ : Let $R$ be an element of the basis

$$
\mathcal{B}=\left\{U_{n}: n \in \omega\right\}
$$

defined in Remark 4.2. The thesis follows from Proposition 4.11, in fact

$$
\llbracket \tau_{f} \in \dot{U}_{n} \rrbracket=\operatorname{Reg}\left(f^{-1}\left[U_{n}\right]\right)
$$

which has meager difference with $f^{-1}\left[U_{n}\right]$. Consider now

$$
R=\bigcup_{i \in \mathcal{I}} U_{i}
$$

where $\mathcal{I}$ is a countable set of indexes. In this case we have that

$$
f^{-1}[R]=\bigcup_{i \in \mathcal{I}} f^{-1}\left[U_{i}\right]
$$

and

$$
\llbracket \tau_{f} \in \dot{R} \rrbracket=\bigvee_{i \in \mathcal{I}} \llbracket \tau_{f} \in \dot{U}_{i} \rrbracket=\operatorname{Reg}(A)
$$

where $A=\bigcup_{i \in \mathcal{I}} \llbracket \tau_{f} \in \dot{U}_{i} \rrbracket$. For each $i \in \mathcal{I}$, the sets $f^{-1}\left[U_{i}\right]$ and $\llbracket \tau_{f} \in \dot{U}_{i} \rrbracket$ have meager difference, thus $f^{-1}[R] \Delta A$ is meager. The proof is therefore concluded because $A \Delta \operatorname{Reg}(A)$ is meager.

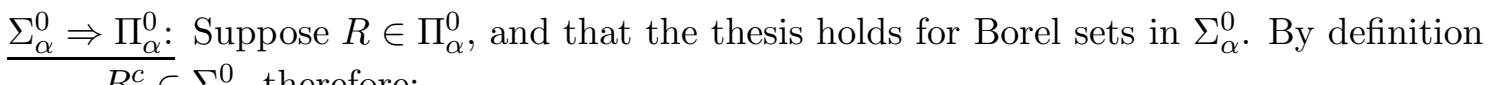
$R^{c} \in \Sigma_{\alpha}^{0}$, therefore:

$$
f^{-1}\left[R^{c}\right] \Delta \llbracket \tau_{f} \in \dot{R}^{c} \rrbracket \text { is meager }
$$

hence

$$
f^{-1}[R] \Delta \llbracket \tau_{f} \in \dot{R} \rrbracket \text { is meager }
$$

$\underline{\Pi_{\alpha}^{0} \Rightarrow \Sigma_{\alpha+1}^{0}}$ : This item can be proved as the second part of the case $\alpha=1$, substituting the $U_{n}$ with Borel sets in $\Pi_{\alpha}^{0}$.

$\Sigma_{\beta}^{0}$ for $\beta$ limit ordinal: If the thesis holds for $\alpha<\beta$, then the proof can be carried similarly to the case $\Pi_{\alpha}^{0} \Rightarrow \Sigma_{\alpha+1}^{0}$.

The Lemma is proved.

This concludes the proof of Theorem 4.6 for the case $Y=\mathbb{C}$.

\section{1. $C(S t(\mathrm{~B})) / G$ and $C^{+}(S t(\mathrm{~B})) / G$ in generic extensions}

The following proposition shows that if we restrict our attention to $V$-generic filters for B then $C(S t(\mathrm{~B}))$ is a family of names large enough to describe all complex numbers of $V[G]$.

Proposition 4.16. Assume $V$ is a model of ZFC, B a complete boolean algebra in $V$ and $G$ a $V$-generic filter in $\mathrm{B}$. Then

$$
C^{+}(S t(\mathrm{~B})) / G \cong C(S t(\mathrm{~B})) / G
$$


Proof. We need to show that for each $f \in C^{+}(S t(\mathrm{~B}))$ we can find an $\tilde{f} \in C(S t(\mathrm{~B}))$ such that

$$
\llbracket f=\tilde{f} \rrbracket \in G
$$

which, by Corollary 4.14, is equivalent to

$$
f^{V[G]}(G)=\tilde{f}^{V[G]}(G)
$$

We denote again

$$
a_{n}=\operatorname{Reg}\left(f^{-1}\left[U_{n}\right]\right) .
$$

Proceeding as in Claim 4.15.1, we can find $m \in \omega$ such that $a_{m} \in G$. For each $H \in \mathcal{O}_{a_{m}}$ we have that

$$
f(H) \in \mathrm{Cl}\left(U_{m}\right)
$$

by Lemma 4.9. We can therefore consider the restriction of $f$ to $\mathcal{O}_{a_{m}}$ (which is clopen) and extend it to a $\tilde{f} \in C(S t(\mathrm{~B}))$ setting it to be constantly 0 on $\mathcal{O}_{\neg a_{m}}$. The implication

$$
f \uparrow_{\mathcal{O}_{a_{m}}^{V}}=\tilde{f} \uparrow_{\mathcal{O}_{a_{m}}^{V}} \Rightarrow f^{V[G]} \uparrow_{\mathcal{O}_{a_{m}}^{V[G]}}=\tilde{f}^{V[G]} \uparrow_{\mathcal{O}_{a_{m}}^{V[G]}}
$$

guarantees the thesis, since $G \in \mathcal{O}_{a_{m}}^{V[G]}$.

\subsection{Proof of Theorem 4.6 for an arbitrary Polish space $Y$}

We outline the proof of Theorem 4.6 for the case of an arbitrary Polish space $Y$. The strategy of the proof is exactly the same for the case $Y=\mathbb{C}$. At some points the corresponding Lemma needs a slightly more elaborate proof, we outline when this is the case. All over this section let

$$
Y=\bigcap_{n \in \mathbb{N}} \bigcup\left\{B_{r_{m n}}\left(q_{m n}\right): m \in \mathbb{N}\right\} \subseteq \mathcal{H}
$$

denote an arbitrary Polish space seen as a $G_{\delta}$ subset of $\mathcal{H}$ and $\left\{U_{n}: n \in \omega\right\}$ denote its basis

$$
\hat{\mathcal{B}}_{Y}=\left\{B_{r}(q) \cap Y: r \in \mathbb{Q}, q \in D\right\}
$$

as done in section 4 .

Lemma 4.17. Assume $f \in V$ is an element of $C^{+}(S t(\mathrm{~B}), Y)$. For $H \in S t(\mathrm{~B})$ we define

$$
\Sigma_{f}^{H}=\left\{\mathrm{Cl}\left(U_{n}\right): \operatorname{Reg}\left(f^{-1}\left[U_{n}\right]\right) \in H\right\}
$$

Then, for $H \in S t(\mathrm{~B})$ such that $f(H) \in Y$, we have:

$$
\{f(H)\}=Y \cap \bigcap \Sigma_{f}^{H},
$$

moreover $\bigcap \Sigma_{f}^{H}$ is always non-empty.

Proof. $\cap \Sigma_{f}^{H}$ is always non-empty, since it is the intersection of a family with the finite intersection property of closed sets of a compact space. The proof that $\left|\cap \Sigma_{f}^{H} \cap Y\right| \leq 1$ if $f(H) \in Y$ runs as the uniqueness part of Lemma 4.9. 
Proposition 4.18. Fix $V$ a transitive model of ZFC and $\mathrm{B} \in V$ a boolean algebra which $V$ models to be complete. Let $f \in C^{+}(S t(\mathrm{~B}), Y)$. For each $n \in \omega$ let

$$
a_{n}=\operatorname{Reg}\left(f^{-1}\left[U_{n}\right]\right)
$$

where $\mathcal{B}_{Y}=\left\{U_{n}: n \in \omega\right\}$ is the fixed countable basis for $Y$. There exists a $\tau_{f} \in Y^{\mathrm{B}}$ such that 5

$$
\llbracket \tau_{f} \in \dot{U}_{n} \rrbracket^{V^{\mathrm{B}}}=a_{n}
$$

for all $n \in \omega$. Moreover any $\tau \in V^{\mathrm{B}}$ such that $\llbracket \tau \in \dot{U}_{n} \rrbracket^{V^{\mathrm{B}}}=a_{n}$ for all $n \in \omega$ is also such that $\llbracket \tau=\tau_{f} \rrbracket^{V^{\mathrm{B}}}=1_{\mathrm{B}}$.

Proof. This proposition has exactly the same proof as the corresponding Proposition 4.11 for $\mathbb{C}$.

Lemma 4.19. Assume $\tau \in Y^{\mathrm{B}}$. Consider

$$
\begin{aligned}
f_{\tau}: S t(\mathrm{~B}) & \rightarrow \mathcal{H} \\
H & \mapsto \sigma_{\tau}^{H}
\end{aligned}
$$

where, given

$$
\Sigma_{\tau}^{H}=\left\{\mathrm{Cl}\left(U_{n}\right): \llbracket \tau \in \dot{U}_{n} \rrbracket^{V^{\mathrm{B}}} \in H\right\}
$$

$\sigma_{\tau}^{H}$ is the unique element in $\bigcap \Sigma_{\tau}^{H}$ if $\Sigma_{\tau}^{H}$ is non-empty. Otherwise $f(H)=\sigma_{\tau}^{H}$ is defined by extending by continuity $f$ on the others $H \in S t(\mathrm{~B})$. The function $f_{\tau}$ belongs to $C^{+}(S t(\mathrm{~B}), Y)$ and $\tau_{f_{\tau}}=\tau$.

Proof. Notice that for all $n \in \omega$

$$
\llbracket \tau \in \bigcup\left\{\dot{B}_{r_{m n}}\left(q_{m n}\right): m \in \mathbb{N}\right\} \rrbracket=1_{\mathrm{B}}
$$

hence for all $n \in \omega$

$$
\left\{\llbracket \tau \in \dot{B}_{r_{m n}}\left(q_{m n}\right) \rrbracket: m \in \omega\right\}
$$

is predense in $\mathrm{B}^{+}$. Seeing each $\llbracket \tau \in \dot{B}_{r_{m n}}\left(q_{m n}\right) \rrbracket$ as a clopen subset of $S t(\mathrm{~B})$, we conclude that

$$
A_{n}=\bigcup\left\{\llbracket \tau \in \dot{B}_{r_{m n}}\left(q_{m n}\right) \rrbracket: m \in \omega\right\}
$$

is open dense in $S t(\mathrm{~B})$ for all $n \in \omega$. Hence $f_{\tau}$ is well defined (and continuous) on the dense $G_{\delta}$ subset of $S t(\mathrm{~B}) \bigcap_{n \in \omega} A_{n}$. Therefore $f$ can be extended by continuity to the whole of $S t(\mathrm{~B})$. The proof of the continuity of $f$ on $\bigcap_{n \in \omega} A_{n}$, and the fact that on $\bigcap_{n \in \omega} A_{n} f$ takes values in $Y$ can be carried as in the corresponding proof of Lemma 4.12,

The proof that

$$
\llbracket f=g \rrbracket^{C^{+}(S t(\mathrm{~B}), Y)}=\llbracket \tau_{f}=\tau_{g} \rrbracket^{V^{\mathrm{B}}}
$$

for any $f, g \in C^{+}(\operatorname{St}(\mathrm{B}), Y)$ is the same as the corresponding proof for equation 1 .

\footnotetext{
${ }^{5}$ If $U_{n}=Y \cap B_{r}(q)$ and $G$ is $V$-generic for B, $\dot{U}_{n}$ denotes the B-name for the elements in the Hilbert cube of $V[G]$ belonging to

$$
\bigcap_{n \in \mathbb{N}} \bigcup\left\{\left(B_{r_{m n}}\left(q_{m n}\right)\right)^{V[G]}: m \in \mathbb{N}\right\} \cap\left(B_{r}(q)\right)^{V[G]},
$$

where $\left(B_{r}(q)\right)^{V[G]}$ is the ball in the Hilbert cube $\mathcal{H}^{V[G]}$ of rational radius $r$ and center $q$ as computed in $V[G]$.
} 


\subsection{Extensions of the boolean isomorphism}

In general any boolean predicate or function on the B-valued model $C^{+}(S t(\mathrm{~B}), Y)$ can be transferred to a corresponding boolean predicate on $Y^{\mathrm{B}}$ using the above isomorphism $f \mapsto \sigma_{f}$.

Definition 4.20. Let $Y$ be a Polish space and B a complete boolean algebra. For any boolean relation $R^{S t(\mathrm{~B})}: C^{+}(S t(\mathrm{~B}), Y)^{n} \rightarrow \mathrm{B}$ (and boolean function $F^{S t(\mathrm{~B})}$ )

$$
R^{\mathrm{B}}\left(\sigma_{1}, \ldots, \sigma_{n}\right)=R^{S t(\mathrm{~B})}\left(f_{\sigma_{1}}, \ldots, f_{\sigma_{n}}\right),
$$

( similarly we can define the boolean function $F^{\mathrm{B}}$ ).

By Theorem 4.6 and Lemma 4.13, we immediately have the following.

Theorem 4.21. Fix a signature

$$
\mathcal{L}=\left\{R_{i}: i \in I\right\} \cup\left\{F_{j}: j \in J\right\} .
$$

and assume that $\left\langle R_{i}^{S t(\mathrm{~B})}: i \in I\right\rangle,\left\langle F_{j}^{S t(\mathrm{~B})}: j \in J\right\rangle$ are boolean interpretations of the signature making $C^{+}(S t(\mathrm{~B}), Y)$ a B-valued model. The map

$$
\begin{aligned}
\Gamma: C^{+}(S t(\mathrm{~B}), Y) & \rightarrow Y^{\mathrm{B}} \\
f & \mapsto \tau_{f}
\end{aligned}
$$

is an isomorphism of the $\mathrm{B}$-valued model

$$
\left\langle C^{+}(S t(\mathrm{~B}), Y), R_{i}^{S t(\mathrm{~B})}: i \in I, F_{j}^{S t(\mathrm{~B})}: j \in J\right\rangle
$$

with the B-valued model

$$
\left\langle Y^{\mathrm{B}}, R_{i}^{\mathrm{B}}: i \in I, F_{j}^{\mathrm{B}}: j \in J\right\rangle .
$$

\subsection{Some further comments on the proof of Theorem 4.6}

One can get a proof of this theorem for the case $Y=\mathbb{C}$ following Jech' 6 results in 5 ] as follows: Jech defines the notion of stonean algebra as an abelian space of (possibly unbounded) normal operators. Stonean algebras are a natural generalization of the notion of commutative $C^{*}$-algebras. Jech proves that:

- The isomorphism type of any complete stonean algebra is determined by the complete boolean algebra given by its space of projections,

- For any complete boolean algebra $\mathrm{B}, \mathbb{C}^{\mathrm{B}}$ and $C^{+}(S t(\mathrm{~B}))$ are complete stonean algebras whose spaces of projections are in both cases isomorphic to $\mathrm{B}$.

Jech's proof that $\mathbb{C}^{\mathrm{B}}$ is a complete stonean algebra exploits the property that $(\mathbb{R},<)$ is a complete linear order in order to give a simple description of the B-names for real numbers of $V^{\mathrm{B}}$, and also the property that any element of a stonean algebra can be decomposed uniquely as the direct sum of its real and imaginary part. The isomorphism between $\mathbb{C}^{\mathrm{B}}$ and $C^{+}(S t(\mathrm{~B}))$ is obtained by showing that the Gelfand transform can be defined also for stonean algebras and yields that any stonean algebra $\mathcal{A}$ is isomorphic to $C^{+}(X)$ where $X$ is

\footnotetext{
${ }^{6}$ This isomorphism of $\mathbb{C}^{\mathrm{B}}$ and $C^{+}(S t(\mathrm{~B}))$ has also been independently proven by Ozawa in [8], but Jech's proof is in our eyes more elegant and informative.
} 
the spectrum of $\mathcal{A}$. Moreover, in case $\mathcal{A}$ is complete, its spectrum $X$ is also homeomorphic to the Stone space of the complete boolean algebra of projections on $\mathcal{A}$. In both arguments there are peculiar properties of $\mathbb{R}$ (being a complete linear order) which are not shared by other Polish spaces $Y$, and of a stonean algebra $\mathcal{A}$ (the characterization of its elements in terms of the involution operation and of its self-adjoint operators) which are not shared by the function spaces $C^{+}(X, Y)$ for $Y \neq \mathbb{C}$. Ozawa's proof relies on the same properties of $\mathbb{R}$ and of commutative algebras of normal operators used in Jech's argument. In particular we do not see any natural pattern to generalize Jech's (or Ozawa's) proof method so to cover also the cases of Theorem 4.6 for a Polish space $Y \neq \mathbb{C}$ other than resorting (as we did) to purely topological characterizations of the properties of Polish spaces. A further comment is in order at this point: we became aware of Jech's and Ozawa's work only after having completed and submitted a first version of this paper.

\section{Generic absoluteness}

We can now show that for any Polish space $Y$ the B-valued models $\left(C^{+}(S t(\mathrm{~B}), Y), R^{S t(\mathrm{~B})}\right)$, with $R$ a Borel (universally Baire) relation on $Y^{n}$, is an elementary superstructure of $(Y, R)$. By Lemma 4.13, whenever $R$ is a Borel relation on $Y^{n}$ with $Y$ Polish, $R^{\mathrm{B}}\left(\sigma_{1}, \ldots, \sigma_{n}\right)=$ $R^{S t(\mathrm{~B})}\left(f_{\sigma_{1}}, \ldots, f_{\sigma_{n}}\right)$ (where $R^{\mathrm{B}}$ is defined as in Def. 4.2). This equality is a special case of the much more general result which can be proved for universally Baire relations.

Definition 5.1 (Feng, Magidor, Woodin [1]). Let $Y$ be a Polish space. $A \subseteq Y^{n}$ is universally Baire if $f^{-1}[A]$ has the Baire property in $Z$ for all continuous $f: Z \rightarrow Y^{n}$ and all compact Hausdorff spaces $Z$.

UB denote the class of universally Baire subsets of $\mathcal{H}$ (or any other Polish space).

Fact 5.2. Let $Y$ be a Polish space. $A \subseteq Y^{n}$ is universally Baire if and only if $f^{-1}[A]$ has the Baire property in $Z$ for all continuous $f: Z \rightarrow Y^{n}$ with $Z$ compact and extremally disconnected.

Proof. We need to prove just one direction, and we prove it as follows. Assume $f: Z \rightarrow$ $Y^{n}$ is continuous for some $Z$ compact Hausdorff but not extremally disconnected. Set $Z^{*}=\operatorname{St}(\operatorname{RO}(Z))$ and define $\pi: Z^{*} \rightarrow Z$ by $\pi(G)=x$ if $x$ is the unique point in $Z$ belonging to

$$
\Sigma^{G}=\bigcap\{\mathrm{Cl}(U): U \in G\} .
$$

The same arguments we encountered in the proof of the isomorphism of $C^{+}(\operatorname{St}(\mathrm{B}))$ with $\mathbb{C}^{\mathrm{B}}$ show that $\pi$ is continuous, open and surjective. In particular $f^{-1}[A]$ has the Baire property in $Z$ iff $g^{-1}[A]$ has the Baire property in $Z^{*}$, where $g=f \circ \pi$.

By [3, Chapter 29, Lemma 5] Borel sets are universally Baire as already observed in Example 3.10, Woodin [7, Theorem 3.4.5, Remark 3.4.7] showed that for any universally Baire set $A$ the theory of $L(\mathbb{R}, A)$ is generically invariant in the presence of class many Woodin cardinals which are a limit of Woodin cardinals, and moreover that these assumptions entail that any $\Sigma_{n}^{1}$-property defines a universally Baire relation. Shoenfield 4 , Lemma 25.20] (or [11, Theorem 3.5.3, Remark 3.5.4] or [13, Lemma 1.2] for a presentation of this result in line with the content of this paper) showed that the $\Sigma_{2}^{1}$-theory of any Polish space $X$ is generically invariant under set forcing. This translates by the results of this paper in the following: 
Theorem 5.3. Assume $\left\langle R_{i}: i \in I\right\rangle$ and $\left\langle F_{j}: j \in J\right\rangle$ are Borel predicates and functions on some Polish space $Y$. Let $X$ be a compact Hausdorff extremally disconnected space and $p \in X$. Then

$$
\left\langle Y, R_{i}: i \in I, F_{j}: j \in J\right\rangle \prec_{\Sigma_{2}}\left\langle C^{+}(X, Y) / p, R_{i}^{X} / p: i \in I, F_{j}^{X} / p: j \in J\right\rangle .
$$

Moreover if we assume the existence of class many Woodin cardinals which are a limit of Woodin cardinals, we can let each $R_{i}$ and $F_{j}$ be arbitrary universally Baire relations and functions, and we have the stronger conclusion that

$$
\left\langle Y, R_{i}: i \in I, F_{j}: j \in J\right\rangle \prec\left\langle C^{+}(X, Y) / p, R_{i}^{X} / p: i \in I, F_{j}^{X} / p: j \in J\right\rangle .
$$

Proof. By Shoenfield's (or Woodin's) theorem we have that for all $\Sigma_{2}^{1}\left(\Sigma_{n}^{1}\right.$ for any $n$ ) properties $\varphi(\vec{x})$ in the parameters $\left\langle R_{i}: i \in I\right\rangle,\left\langle F_{j}: j \in J\right\rangle$ with each $R_{i}, F_{j}$ Borel (universally Baire) the following are equivalent:

- $\varphi(\vec{r})$ holds in $\left\langle Y, R_{i}: i \in I, F_{j}: j \in J\right\rangle$,

- $\llbracket \varphi(\vec{r}) \rrbracket=1_{\mathrm{B}}$ in $\mathbb{C}^{\mathrm{B}}$ for some complete boolean algebra $\mathrm{B}$,

- $\llbracket \varphi(\vec{r}) \rrbracket=1_{\mathrm{B}}$ in $\mathbb{C}^{B}$ for all complete boolean algebras $\mathrm{B}$.

Since $X$ is compact Hausdorff and extremally disconnected, $\mathrm{CL}(X)$ is a complete boolean algebra and $X$ is homeomorphic to $\operatorname{St}(\mathrm{CL}(X))$. By Theorem 4.21 $C^{+}(X, Y)$ and $Y^{\mathrm{B}}$ are isomorphic B-valued models. In particular $C^{+}(X, Y)$ is full. By the first two equivalent items we get that $\llbracket \varphi(\vec{r}) \rrbracket^{C^{+}(X, Y)}=1_{\mathrm{B}}$ in $C^{+}(X, Y)$ if and only if $\varphi(\vec{r})$ holds in $Y$. Since the above holds for all relevant properties $\varphi$, we can apply Loś's theorem to the full B-valued model $C^{+}(X, Y)$ in the point (ultrafilter) $p$ to conclude that

$$
\left\langle Y, R_{i}: i \in I, F_{j}: j \in J\right\rangle \prec{ }_{\left(\Sigma_{2}\right)}\left\langle C^{+}(X, Y) / p, R_{i}^{X} / p: i \in I, F_{j}^{X} / p: j \in J\right\rangle .
$$

Following Takeuti's ideas, we remark that these results suggest the following "original" proof strategy to be applied in an algebraic geometric context rather than in an operator algebra context (as already done by Takeuti and others). Prove that a certain problem regarding for example complex numbers and analytic functions has a solution in some forcing extension. Then argue that its solution can be formalized as a first order property of the structure $C^{+}(X) / p$. Conclude using elementarity that the solution of the problem for the complex numbers is really the one computed in $C^{+}(X) / p$. We have already successfully applied the above strategy to prove a result related to Schanuel's conjecture in number theory (unfortunately for us already proved by other means): the interested reader is referred to [12].

\section{Acknowledgements}

The second author acknowledges support from the PRIN2012 Grant "Logic, Models and Sets" (2012LZEBFL), and the Junior PI San Paolo grant 2012 NPOI (TO-Call1-20120076). This research was completed whilst the second author was a visiting fellow at the Isaac Newton Institute for Mathematical Sciences in the programme "Mathematical, Foundational and Computational Aspects of the Higher Infinite" (HIF) funded by EPSRC grant EP/K032208/1. 


\section{REFERENCES}

[1] Qi Feng, Menachem Magidor, and Hugh Woodin, Universally Baire sets of reals, Set theory of the continuum (Berkeley, CA, 1989) (H. Judah, W. Just, and H. Woodin, eds.), Math. Sci. Res. Inst. Publ., vol. 26, Springer, New York, 1992, pp. 203-242. MR MR1233821 (94g:03095)

[2] Michael P. Fourman, Christopher J. Mulvey, and Dana S. Scott (eds.), Applications of sheaves, Lecture Notes in Mathematics, vol. 753, Springer, Berlin, 1979. MR 555538

[3] Steven Givant and Paul Halmos, Introduction to Boolean algebras, Undergraduate Texts in Mathematics, Springer, New York, 2009. MR 2466574 (2009j:06001)

[4] Thomas Jech, Set theory, Springer Monographs in Mathematics, Springer-Verlag, Berlin, 2003, The third millennium edition, revised and expanded. MR 1940513 (2004g:03071)

[5] Thomas J. Jech, Abstract theory of abelian operator algebras: an application of forcing, Trans. Amer. Math. Soc. 289 (1985), no. 1, 133-162. MR 779056

[6] Alexander S. Kechris, Classical descriptive set theory, Graduate Texts in Mathematics, vol. 156, Springer-Verlag, New York, 1995. MR 1321597 (96e:03057)

[7] Paul B. Larson, The stationary tower, University Lecture Series, vol. 32, American Mathematical Society, Providence, RI, 2004, Notes on a course by W. Hugh Woodin. MR 2069032

[8] Masanao Ozawa, A classification of type I $A W^{*}$-algebras and Boolean valued analysis, J. Math. Soc. Japan 36 (1984), no. 4, 589-608. MR 759416

[9] Helena Rasiowa and Roman Sikorski, The mathematics of metamathematics, Monografie Matematyczne, no. 41, Polish Scientific Publishers, 1963.

[10] Gaisi Takeuti, Two applications of logic to mathematics, Iwanami Shoten, Publishers, Tokyo; Princeton University Press, Princeton, N.J., 1978, Kanô Memorial Lectures, Vol. 3, Publications of the Mathematical Society of Japan, No. 13. MR 0505474

[11] Andrea Vaccaro, $C^{*}$-algebras and B-names for complex numbers, (2015), Unpublished Master Thesis, (https://etd.adm.unipi.it/t/etd-08302015-193406/).

[12] Matteo Viale, Forcing the truth of a weak form of Schanuel's conjecture, (2015), To appear in Confluentes Mathematici.

[13] _ Martin's maximum revisited, Arch. Math. Logic 55 (2016), no. 1-2, 295-317. MR 3453587

[14] W. Hugh Woodin, Supercompact cardinals, sets of reals, and weakly homogeneous trees, Proc. Nat. Acad. Sci. U.S.A. 85 (1988), no. 18, 6587-6591. MR 959110

[15] — The axiom of determinacy, forcing axioms, and the nonstationary ideal, de Gruyter Series in Logic and its Applications, vol. 1, Walter de Gruyter \& Co., Berlin, 1999. MR 1713438 